\title{
Substituent Effects on the Thermal Decomposition of Phosphate Esters on Ferrous Surfaces
}

James P. Ewen, ${ }^{1,+,{ }^{*}}$ Carlos Ayestarán Latorre,,${ }^{1,2,+}$ Chiara Gattinoni, ${ }^{3}$ Arash Khajeh, ${ }^{4}$ Joshua D. Moore,${ }^{5}$ Joseph E. Remias, ${ }^{5}$ Ashlie Martini, ${ }^{4}$ and Daniele Dini ${ }^{1}$

1 - Department of Mechanical Engineering, Imperial College London, London, SW7 2AZ, U.K.

${ }^{2}$ - Department of Materials, Imperial College London, London, SW7 2AZ, U.K.

2 - Department of Materials, ETH Zürich, Zürich 8092, Switzerland.

4 - Department of Mechanical Engineering, University of California Merced, Merced, California 95343, U.S.A.

5 - Afton Chemical Corporation, Richmond, Virginia 23219, U.S.A.

* - Corresponding author email: j.ewen@imperial.ac.uk

+ - J.P.E. and C.A.L. contributed equally to this work.

\section{Abstract}

Phosphate esters have a wide range of industrial applications, for example in tribology where they are used as vapour phase lubricants and antiwear additives. An atomic-level understanding of phosphate ester tribofilm formation mechanisms is required to improve their tribological performance. A process of particular interest is the thermal decomposition of phosphate esters on steel surfaces, since this initiates polyphosphate film formation. In this study, reactive force field (ReaxFF) molecular dynamics (MD) simulations are used to study the thermal decomposition of phosphate esters with different substituents on several ferrous surfaces. The ReaxFF parameterisation was validated for a representative system using density functional theory (DFT) calculations. During the MD simulations on $\mathrm{Fe}_{3} \mathrm{O}_{4}(001)$ and $\alpha-$ $\mathrm{Fe}(110)$, chemisorption interactions between the phosphate esters and the surfaces occur even at room temperature, and the number of molecule-surface bonds increases as the temperature increases from 300 to $1000 \mathrm{~K}$. Conversely, on hydroxylated, amorphous $\mathrm{Fe}_{3} \mathrm{O}_{4}$, most of the molecules are physisorbed and some desorption occurs at high temperature. Thermal decomposition rates were much higher on $\mathrm{Fe}_{3} \mathrm{O}_{4}(001)$ and particularly $\alpha-\mathrm{Fe}(110)$ compared to hydroxylated, amorphous $\mathrm{Fe}_{3} \mathrm{O}_{4}$. This suggests that water passivates ferrous surfaces and inhibits phosphate ester chemisorption, decomposition, and ultimately polyphosphate film formation. For the alkyl phosphates, thermal decomposition proceeds mainly through $\mathrm{C}-\mathrm{O}$ and $\mathrm{C}-\mathrm{H}$ cleavage on $\mathrm{Fe}_{3} \mathrm{O}_{4}(001)$. Aryl phosphates show much higher thermal stability, and decomposition on $\mathrm{Fe}_{3} \mathrm{O}_{4}(001)$ only occurs through $\mathrm{P}-\mathrm{O}$ and $\mathrm{C}-\mathrm{H}$ 
cleavage, which require very high temperature. The onset temperature for $\mathrm{C}-\mathrm{O}$ cleavage on $\mathrm{Fe}_{3} \mathrm{O}_{4}(001)$ increases as: tertiary alkyl < secondary alkyl < primary linear alkyl $\approx$ primary branched alkyl < aryl. This order is consistent with experimental observations for the thermal stability of antiwear additives with similar substituents. The simulation results clarify a range of surface and substituent effects on the thermal decomposition of phosphate esters on steel that should be helpful for the design of new molecules with improved tribological performance.

\section{Introduction}

Rational design of lubricant additive molecules could increase the energy efficiency and reliability of engineering systems by reducing friction and wear inside machine components. ${ }^{1}$ However, this requires a detailed understanding of the molecular-scale processes (adsorption, dissociation, and polymerisation) that drive tribological behaviour (friction and wear) at the macroscale. ${ }^{2}$ One important additive is zinc dialkyldithiophosphate (ZDDP), which remains ubiquitous in lubricant formulations to mitigate wear inside internal combustion engines. ${ }^{3}$ However, ZDDP contains high levels of zinc, sulfur, and phosphorus, which are known to degrade exhaust aftertreatment devices. ${ }^{4}$ Consequently, zinc-free, low-sulphur, and lowphosphorus alternatives to ZDDP have long been sought. ${ }^{5}$ Some promising examples include phosphate esters and thiophosphate esters. ${ }^{6}$ Alkyl phosphates, such as tri( $n$-butyl)phosphate (TNBP), are already used as additives in gear lubricants and industrial lubricants. ${ }^{7}$ Moreover, aryl phosphates, such as tri $(m$-cresyl) phosphate (TCP), are included in most aviation lubricant formulations. ${ }^{8}$ Both TNBP and TCP have also been used as vapour phase lubricants (VPLs) for tribological systems that operate at very high temperature $(>700 \mathrm{~K}){ }^{9}$

TCP is the most widely studied phosphate ester antiwear additive.$^{5}$ In 1940 , Beeck et al. ${ }^{10}$ suggested that the ability of TCP to reduce wear of steel surfaces originated from chemical polishing via the formation of iron phosphide. However, later work indicated that the major component of the tribofilms formed from TCP was iron phosphate, which acted as a protective layer rather than polishing the steel surfaces. ${ }^{11-13}$ It has also been shown that organic polyphosphates $^{14}$ or iron polyphosphates ${ }^{15,16}$ are eventually formed from TCP inside tribological contacts and are ultimately responsible for its antiwear performance on steel surfaces. ${ }^{8}$ At high temperature, TCP can form relatively thick $(60-100 \mathrm{~nm})$ thermal films on steel surfaces, ${ }^{17}$ but thinner tribofilms are usually formed inside rubbing contacts. ${ }^{5}$

The formation of iron phosphate/polyphosphate films requires decomposition of the phosphate ester molecules, more specifically removal of the alkyl or aryl substituents. ${ }^{6}$ Under the ultrahigh vacuum (UHV) conditions relevant to VPL, this proceeds via molecular adsorption, followed by C-O and/or P-O cleavage to facilitate the formation of a network of P-O bonds. ${ }^{15,16}$ There are several hypotheses concerning the alkyl/aryl substituent removal mechanism from 
phosphate ester antiwear additives on steel surfaces. These include that: (a) intact phosphate esters adsorb and then thermally decompose, (b) intact molecules adsorb and decompose by nucleophilic attack by other surface-adsorbed species (e.g., $\mathrm{O}^{2-}$ or $\mathrm{OH}^{-}$), or (c) phosphate esters act as a reservoir for phosphoric acid which is formed by prior hydrolysis in base oil solution. ${ }^{8}$ There have been contradictory reports regarding the role of water on TCP antiwear performance; it was initially suggested that hydrolysed TCP shows lower wear than intact $\mathrm{TCP},{ }^{18}$ but subsequent studies have shown that the presence of water in TCP-containing lubricants increased wear. ${ }^{19}$

The effects of different substituents on the tribological performance of phosphate esters on steel surfaces has also been investigated. Aryl phosphates are known to have higher thermal stability than alkyl phosphates; ${ }^{20}$ however, there have been conflicting reports of their relative antiwear performance on steel surfaces. While the use of additives with lower thermal stability could ensure that protective tribofilms are formed on sliding surfaces more quickly, this could also result in rapid depletion of the additive, particularly under severe conditions. ${ }^{21}$ In their pure form, aryl phosphates have shown improved wear resistance compared to alkyl phosphates under extreme pressure (EP) conditions; ${ }^{22}$ however, the opposite trend has been reported when they are dissolved in a base oil. ${ }^{23,24}$ There is some disagreement in the literature regarding the dominant bond breaking processes during the thermal decomposition of phosphate esters at high temperature $(>700 \mathrm{~K})$ on ferrous surfaces. Some studies have suggested that alkyl phosphates (e.g., TNBP) decompose mainly via C-O bond cleavage, ${ }^{25,26}$ while others have suggested that P-O cleavage dominates ${ }^{27}$ For aryl phosphates (e.g., TCP), it is widely agreed that $\mathrm{P}-\mathrm{O}$ cleavage dominates..$^{25,26}$

Molecular dynamics (MD) simulations and density functional theory (DFT) calculations have also given unique insights into the decomposition mechanisms of phosphate esters. ${ }^{28}$ For example, static DFT calculations have been used to study the adsorption and dissociation of TCP on iron surfaces. ${ }^{29}$ The calculations showed that the mechanisms (a), (b), and (c) described above all have at least one exothermic reaction pathway. ${ }^{29}$ In addition to static calculations, ab initio molecular dynamics (AIMD) simulations have also been used to investigate the dissociation of tri(methyl)phosphate, ${ }^{30}$ tri(methyl)phosphite, ${ }^{31}$ and tri( $n$ butyl)phosphite ${ }^{32}$ confined between iron surfaces where load and sliding are applied. The AIMD simulations have given insight into the dissociation pathways of organophosphorus molecules inside tribological contacts. However, the high computational expense of AIMD simulations means that studies are limited in terms of the accessible length and time scales. Alternatively, empirical approaches such as classical MD simulations using many-body, bond order potentials can be applied. One popular example is the Reactive Force Field (ReaxFF), ${ }^{33}$ which is several orders of magnitude more computationally efficient than ab initio 
approaches ${ }^{34}$ ReaxFF requires careful parameterisation to reproduce quantities calculated using $a b$ initio methods for a representative training set to ensure accuracy. This parameterisation process has now been conducted for an extensive range of systems and conditions, and in many cases provides accuracy close to that obtained using ab initio methods. ${ }^{35}$ For the study of phosphate esters, a ReaxFF parameterisation has recently been developed for $\mathrm{C} / \mathrm{H} / \mathrm{O} / \mathrm{Fe} / \mathrm{P}$-containing systems. ${ }^{36}$ It has been applied to study the thermal decomposition of TCP on hydroxylated, amorphous iron oxide surfaces. ${ }^{36,37}$

In this study, the thermal decomposition of phosphate esters on ferrous surfaces is investigated using a combination of ReaxFF MD simulations and DFT calculations. In the MD simulations, phosphate esters with a wide range of alkyl and aryl substituents are compared on nascent and passivated ferrous surfaces. DFT calculations are used to verify the ReaxFF parameterisation for a representative molecule-surface combination. The observed differences between the various surfaces and molecules with different substituents have important implications for the design of new phosphate ester molecules for use as VPLs and antiwear additives. 


\section{Methodology}

\section{System Setup}

Phosphate triesters with five different substituents were compared: (i) primary linear alkyl, tri( $n$ butyl)phosphate, TNBP; (ii) primary branched alkyl, tri(i-butyl)phosphate (TIBP); (iii) secondary alkyl, tri(s-butyl)phosphate (TSBP); (iv) tertiary alkyl, tri(t-butyl)phosphate (TTBP); and $(\mathrm{v})$ aryl, tri $(m$-cresyl)phosphate (TCP). The molecular structures of the five phosphate esters considered are shown in Fig. 1.

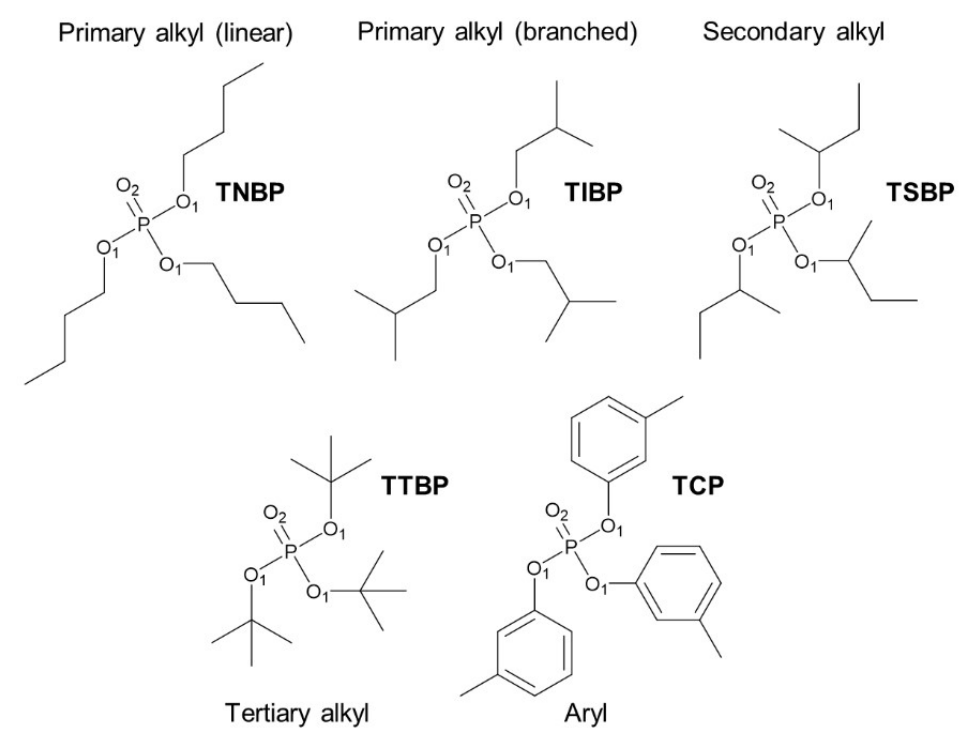

Figure 1. Molecular structures of phosphate esters considered: TNBP (primary linear alkyl), TIBP (primary branched alkyl), TSBP (secondary alkyl), TTBP (tertiary alkyl), and TCP (aryl). Oxygen atoms in $\mathrm{P}-\mathrm{O}$ bonds are labelled $\mathrm{O}_{1}$, while those in $\mathrm{P}=\mathrm{O}$ bonds are labelled $\mathrm{O}_{2}$.

The most appropriate surface for MD simulations involving steel specimens for tribological systems is still debated. For example, $\mathrm{FeO},{ }^{38} \mathrm{Fe}_{2} \mathrm{O}_{3},{ }^{38,39} \mathrm{Fe}_{3} \mathrm{O}_{4},{ }^{38}$ and hydroxylated, amorphous $\mathrm{Fe}_{3} \mathrm{O}_{4}{ }^{36,37}$ have all been used to represent steel substrates in recent MD studies. This is primarily due to the plethora of passivating iron oxides and surface terminations which could be formed. ${ }^{40}$ In aqueous environments, X-ray photoelectron spectroscopy (XPS) experiments have shown that the outermost layer of carbon steels consists mostly of hydroxylated $\mathrm{Fe}_{2} \mathrm{O}_{3} \cdot{ }^{41}$ Less is known about the surface oxides or hydroxides formed on the steels used in machine components lubricated by hydrocarbon-based fluids. Evans et al. performed XPS and transmission electron microscope (TEM) analysis of the wear tracks 
formed during tribology experiments using bearing steel lubricated with a hydrocarbon base $\mathrm{oil}^{42}$ and $\mathrm{P}$-containing additives dissolved in base oil. ${ }^{43}$ The XPS showed that $\mathrm{FeO}, \mathrm{Fe}_{2} \mathrm{O}_{3}$, and $\mathrm{Fe}_{3} \mathrm{O}_{4}$ were all present at the surface, while TEM indicated that the surface was heterogeneous, consisting of local crystalline and amorphous regions. ${ }^{42,43}$ Tests under boundary lubrication conditions (where more substantial temperature rises are expected) contained more amorphous material compared to tests under mixed lubrication conditions. ${ }^{42,43}$ Furthermore, lubricants can often become contaminated with water, ${ }^{44}$ which is likely to adsorb and dissociate on iron oxide surfaces, ultimately leading to hydroxyl formation. ${ }^{45}$

MD simulations are generally limited to the nanometre length scales, ${ }^{28}$ so it is not feasible to investigate the heterogeneous surfaces observed experimentally. ${ }^{42,43}$ In this study, we compare behaviour on three particularly relevant substrates: (i) nascent $\alpha-\mathrm{Fe}$, which could be maintained under UHV conditions ${ }^{25,26}$ or exposed during rubbing in conventional tribology experiments; ${ }^{46}$ (ii) crystalline magnetite $\left(\mathrm{Fe}_{3} \mathrm{O}_{4}\right)$, which has been observed on steel samples from tribology experiments; ${ }^{42,43}$ and (iii) amorphous magnetite with surface hydroxylation (a$\mathrm{Fe}_{3} \mathrm{O}_{4}-\mathrm{OH}$ ), which could be formed due to large temperature rises ${ }^{42,43}$ and water contamination. ${ }^{45}$ We considered the $\alpha-\mathrm{Fe}(110)^{47}$ and $\mathrm{Fe}_{3} \mathrm{O}_{4}(001)^{40}$ planes since they show high thermodynamic stability. For $\mathrm{Fe}_{3} \mathrm{O}_{4}(001)$, the so-called B-layer termination is chosen, which is stable under oxygen-rich conditions. ${ }^{48}$ The thermal decomposition of all of the phosphate esters (shown in Fig. 1) was investigated on $\mathrm{Fe}_{3} \mathrm{O}_{4}(001)$. The primary linear alkyl (TNBP) and secondary alkyl (TSBP) molecules were also compared on $\alpha-\mathrm{Fe}(110)$ and a$\mathrm{Fe}_{3} \mathrm{O}_{4}-\mathrm{OH}$.

All of the systems were constructed using the Materials and Processes Simulations (MAPS) platform from Scienomics SARL. The substrates had approximate dimensions of $x=5.0 \mathrm{~nm}$, $y=5.0 \mathrm{~nm}$, and $z=1.5 \mathrm{~nm} .3 .5 \mathrm{~nm}$ of vacuum was added above the substrate in the $z-$ direction. Sixteen phosphate ester molecules were added approximately $0.3 \mathrm{~nm}$ from the surface in the $z$-direction, corresponding to a surface coverage of 0.6 molecules $\mathrm{nm}^{-2}$. We are not aware of adsorption experiments for phosphate esters from non-aqueous solvents. However, adsorption experiments for $n$-butyl, $i$-butyl, and $s$-butyl ZDDPs on iron oxide from $n$ hexadecane suggested a plateau coverage of approximately 0.5 molecules $\mathrm{nm}^{-2}$ for all of the butyl isomers. ${ }^{49}$ The alkyl phosphate systems (TNBP, TIBP, TSBP, TTBP) contain a total of $192 \mathrm{C}$ atoms, $432 \mathrm{H}$ atoms, $64 \mathrm{O}$ atoms, and $16 \mathrm{P}$ atoms while the aryl phosphate system (TCP) contains $336 \mathrm{C}$ atoms, $336 \mathrm{H}$ atoms, $64 \mathrm{O}$ atoms, and $16 \mathrm{P}$ atoms (not including the surface atoms). To sample a wider configurational space, half of the phosphate ester molecules were initially orientated with $\mathrm{O}$ atoms in the $\mathrm{P}=\mathrm{O}$ bonds pointed towards the surface, and half with them pointing away. ${ }^{36}$ Some representative systems for the thermal decomposition simulations are shown in Fig. 2. 

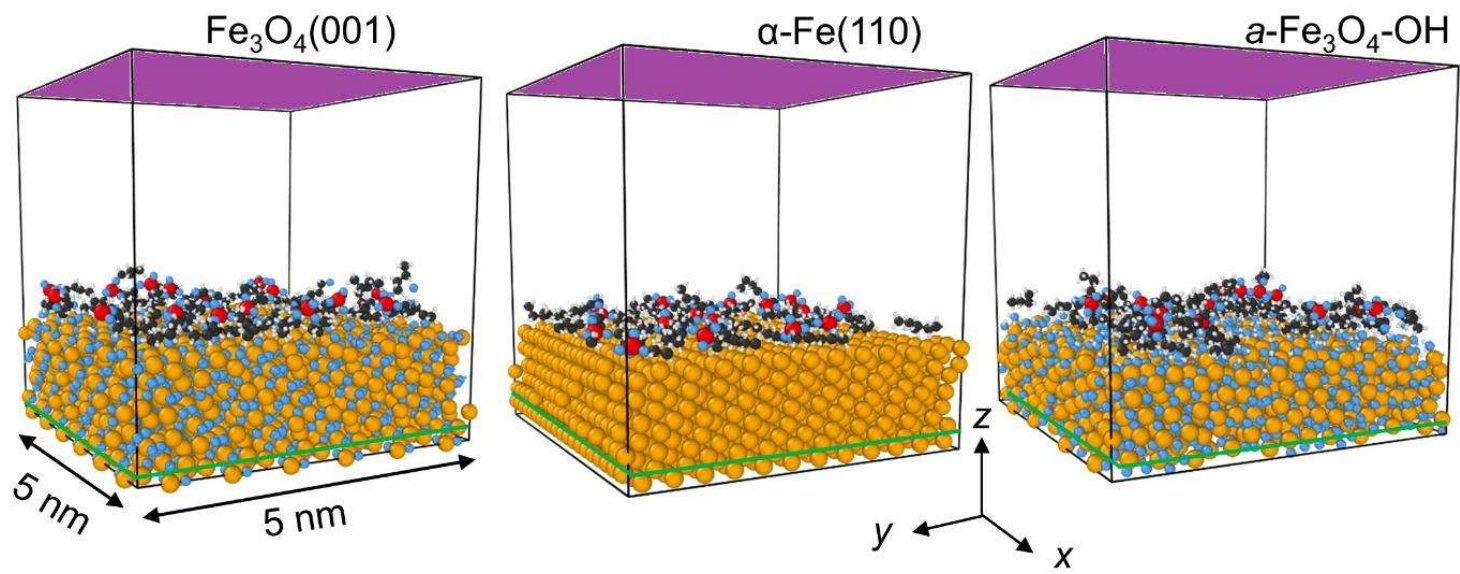

Figure 2. Snapshots of representative systems TNBP on $\mathrm{Fe}_{3} \mathrm{O}_{4}(001), \alpha-\mathrm{Fe}(110)$, and $a-\mathrm{Fe}_{3} \mathrm{O}_{4}-$ $\mathrm{OH}$ after energy minimisation and before equilibration. Visualised with OVITO: ${ }^{50} \mathrm{Fe}$ atoms shown in orange, $\mathrm{O}$ in blue, $\mathrm{P}$ in red, $\mathrm{C}$ in black, and $\mathrm{H}$ in white. Atoms below the green line are frozen. The purple shaded area is a reflective boundary in the $x y$ plane at the top of the simulation cell. Note that all of the molecules are intact at this stage, even though some appear to be broken due to the periodic boundary conditions in $x$ and $y$ directions.

\section{Simulation Details}

The large atomic/molecular massively parallel simulator (LAMMPS) software was used for the MD simulations. ${ }^{51}$ Velocity Verlet integration was used with a time step of $0.25 \mathrm{fs} .{ }^{36}$ The bottom atomic layer of the substrate was frozen, and periodic boundary conditions were applied in the $x$ and $y$ directions. The temperature was controlled using a Nosé-Hoover thermostat ${ }^{52,53}$ with a damping parameter of $25 \mathrm{fs}$, which was applied to all non-frozen atoms. A reflective boundary was added in the $x y$ plane at the top of the simulation cell $(z=5.0 \mathrm{~nm})$ to prevent desorbed species from leaving the simulation cell (see Fig. 2). ${ }^{37}$

\section{Force Field}

All of the MD simulations used the same ReaxFF parameters for $\mathrm{C} / \mathrm{H} / \mathrm{O} / \mathrm{Fe} / \mathrm{P}$ interactions as were employed by Khajeh et al. ${ }^{36}$ ReaxFF is a bond order-based force field that was originally developed by van Duin et al. ${ }^{33}$ to study the dissociation and reactions of small hydrocarbon molecules. The version of ReaxFF implemented in LAMMPS uses the functional form which was outlined by Chenoweth et al. ${ }^{54}$ and was fully described by Aktulga et al. ${ }^{55}$ The general form of ReaxFF is given by: ${ }^{35}$

$E_{\text {system }}=E_{\text {bond }}+E_{\text {over }}+E_{\text {angle }}+E_{\text {tors }}+E_{v a w}+E_{\text {coulomb }}+E_{\text {specific }}$ 
where $E_{b o n d}$ is a continuous function of interatomic distance, describing the energy associated with bond formation (including $\sigma, \pi$, and $\pi-\pi$ contributions). $E_{\text {angle }}$ and $E_{\text {tors }}$ are the energies associated with three-body angle and four-body torsional angle strain respectively. $E_{o v e r}$ is an energy penalty to prevent over-coordination of atoms and is based on atomic valence rules. $E_{\text {Coulomb }}$ and $E_{v d W}$ represent the electrostatic and dispersive interactions between all atoms in the system, irrespective of their connectivity and bond order. $E_{\text {specific }}$ represents systemspecific terms required to capture properties particular to the system of interest, such as lonepairs, conjugation, and hydrogen bonding. ${ }^{35}$ The point charges on the atoms vary dynamically during the MD simulation and are calculated using the charge equilibration (Qeq) method. ${ }^{55-}$ 57

In the ReaxFF parameter file used in the current study (see Supporting Information), the $\mathrm{Fe} / \mathrm{P} / \mathrm{O}$ ReaxFF parameters were developed by Khajeh et $a{ }^{36}{ }^{36}$ The parameters for $\mathrm{Fe} / \mathrm{O} / \mathrm{H}$ were originally developed by Aryanpour et al., ${ }^{58} \mathrm{Fe} / \mathrm{C}$ by Zou et al., ${ }^{59} \mathrm{P} / \mathrm{O} / \mathrm{C} / \mathrm{H}$ by Verlackt et al. ${ }^{60}$, and $\mathrm{C} / \mathrm{H} / \mathrm{O}$ by Chenoweth et al. ${ }^{54}$ The ReaxFF parameters for $\mathrm{Fe} / \mathrm{O} / \mathrm{H}^{58}$ have been shown to accurately reproduce the experimental lattice parameters for $\alpha-\mathrm{Fe}$ (within $1 \%$ ), ${ }^{59} \alpha-\mathrm{Fe}_{2} \mathrm{O}_{3}$ (within $4 \%$ ), ${ }^{39}$ and $\mathrm{Fe}_{3} \mathrm{O}_{4}$ (within $3 \%$ ). ${ }^{58}$ They have also been shown to perform favourably compared to other many-body force fields in describing the mechanical properties of $\alpha$-Fe. ${ }^{61}$ MD simulations using ReaxFF have been successfully employed to study the thermal decomposition of a wide range of molecules; from hydrazines, ${ }^{62}$ to methylnaphthalene derivatives, ${ }^{63}$ and hydrocarbons adsorbed on nickel surfaces. ${ }^{64}$ The $\mathrm{C} / \mathrm{H} / \mathrm{O} / \mathrm{Fe}$ parameters used in this study have also shown good agreement with experiment in MD simulations of heterogeneous catalysis, for example the methanation of the carbon monoxide by $\mathrm{Fe}(100)^{65}$, the dissociative adsorption of formaldehyde on $\mathrm{Fe}(100),{ }^{66}$ and the oxidation of butane on $\mathrm{Cr}_{2} \mathrm{O}_{3}$ in the presence of $\mathrm{FeS}_{2}{ }^{67}$ The ReaxFF parameter set has previously been applied to study the thermal decomposition of TCP on a- $\mathrm{Fe}_{3} \mathrm{O}_{4}-\mathrm{OH}$ surfaces $^{36}$ as well as TCP interactions with nanodiamonds on the same surface. ${ }^{37}$

\section{DFT Validation}

The ReaxFF parameterisation was validated using ab initio methods for the adsorption of an intact TNBP molecule on $\alpha-\mathrm{Fe}(110)$. The energy changes for $\mathrm{C}-\mathrm{O}$ and $\mathrm{P}-\mathrm{O}$ dissociation for this molecule-surface combination were also investigated. Spin-polarised DFT calculations were performed using the Vienna ab initio simulation program (VASP) ${ }^{68-70}$ with the projector augmented wave (PAW) pseudopotentials. ${ }^{71}$ Tests were performed with both the PerdewBurke-Ernzerhof (PBE) exchange-correlation functional ${ }^{72}$ and the optB86b-vdW nonlocal correlation functional, ${ }^{73}$ which explicitly treats van der Waals interactions. Unlike some previous molecular adsorption studies, ${ }^{74,75}$ similar adsorption energies were obtained with both functionals. The optB86b-vdW functional was used for the production calculations. The 
plane-wave cutoff energy was set to $550 \mathrm{eV}$. The convergence criteria for the total energy in the self-consistent cycle was $10^{-6} \mathrm{eV}$, while for the ionic forces during geometry optimization it was $10^{-4} \mathrm{eV} \AA^{-1}$. The Monkhorst-Pack scheme ${ }^{76}$ was used for k-point sampling with a $3 \times 2$ $\times 1 \mathrm{k}$-mesh for a supercell with an $\alpha-\mathrm{Fe}(110)$ slab with dimensions of $x=1.2 \mathrm{~nm}, y=1.7 \mathrm{~nm}$, $z=2.4 \mathrm{~nm}$. A single TNBP molecule was placed $0.3 \mathrm{~nm}$ above the slab, which corresponds to a similar surface coverage $\left(0.5 \mathrm{~nm}^{-2}\right)$ to the ReaxFF MD simulations. A dipole correction was added in the $z$-direction. ${ }^{77} 1.5 \mathrm{~nm}$ of vacuum space was maintained above the Fe surface. The lattice constant and the bottom layer of Fe atoms was fixed at the bulk crystal structure during the geometry optimisation.

The adsorption energy, $E_{a d s}$, was calculated using the expression:

$E_{a d s}=E_{s y s}-\left(E_{s l a b}+E_{m o l}\right)$

where $E_{\text {sys }}$ is the total energy of the adsorbed complex, $E_{\text {slab }}$ is the total energy of the fully relaxed substrate, and $E_{m o l}$ is the total energy of the relaxed isolated molecule. For the validation procedure, the $E_{a d s}$ values of both intact and dissociated TNBP molecules adsorbed on $\alpha-\mathrm{Fe}(110)$ were compared following geometry optimisation using either ReaxFF or DFT. The most stable intact and dissociated structures were identified through energy minimisation of ten starting structures with different molecule orientations using ReaxFF. The most stable structures were then geometry optimised using DFT.

Nudged elastic band (NEB) calculations with the climbing image scheme ${ }^{78}$ were used to calculate the minimum energy path along the potential energy surface for $\mathrm{C}-\mathrm{O}$ and $\mathrm{P}-\mathrm{O}$ bond dissociation. The NEB calculations were performed in LAMMPS ${ }^{51}$ with the same ReaxFF parameters used in the MD simulations. ${ }^{36}$ The energies obtained from the NEB calculations using ReaxFF were compared to single-point DFT calculations of the same structures.

\section{a- $\mathrm{Fe}_{3} \mathrm{O}_{4}-\mathrm{OH}$ Surface Preparation}

The $a-\mathrm{Fe}_{3} \mathrm{O}_{4}$ surface was produced by annealing a slab of crystalline $\mathrm{Fe}_{3} \mathrm{O}_{4}$. The temperature was increased from 300 to $4000 \mathrm{~K}$ over $25 \mathrm{ps}$, held at $4000 \mathrm{~K}$ for $125 \mathrm{ps}$, and then quenched back to $300 \mathrm{~K}$ over $500 \mathrm{ps}$. Before amorphisation, the Fe coordination number was 4 (37\%) or $6(63 \%)$, as expected for $\mathrm{Fe}_{3} \mathrm{O}_{4}$. Following amorphisation, the majority of the Fe atoms had a coordination number of 4 (78\% in the bulk and $80 \%$ on the surface), with the remaining $\mathrm{Fe}$ atoms having coordination numbers of 1 ( $0.2 \%$ bulk, $3 \%$ surface), 2 (3\% bulk, $8 \%$ surface), 3 (17\% bulk and $8 \%$ surface), and 5 ( $1 \%$ bulk, $0 \%$ surface), ${ }^{36}$ which is indicative of an amorphous substrate.

Finally, to hydroxylate the surface, 300 water molecules were placed over the $a-\mathrm{Fe}_{3} \mathrm{O}_{4}$ substrate, and the temperature was increased to $700 \mathrm{~K}$ to accelerate hydroxylation. The 
simulation was run for $500 \mathrm{ps}$, until the potential energy was stable. After this stage, any atoms from intact or dissociated water molecules that were not covalently bonded to the surface were removed. ${ }^{36}$ At the end of this process, the hydroxyl group density on the surface was $8.3 \mathrm{~nm}^{-2}$, which is within the experimentally observed range $\left(7-20 \mathrm{~nm}^{-2}\right)$ for $\mathrm{Fe}_{3} \mathrm{O}_{4}{ }^{79}$

\section{Simulations}

Initially, the energy of each system was minimised using the conjugate gradient method, followed by equilibration for $0.2 \mathrm{~ns}$ at $300 \mathrm{~K}$. Then, the temperature was linearly increased from 300 to $1000 \mathrm{~K}$ over $1.4 \mathrm{~ns}$ (heating rate of $0.5 \mathrm{~K} \mathrm{ps}^{-1}$ ). ${ }^{37}$ The selected temperature range is representative of that experienced by phosphate esters when they are used as VPLs under UHV conditions ${ }^{9}$ and antiwear additives under EP conditions. ${ }^{80}$ Previous ReaxFF studies of hydrazine molecules showed that, as the heating rate was increased from 50 to $200 \mathrm{~K} \mathrm{ps}^{-1}$, the onset temperature for decomposition increased from 1350 to $1690 \mathrm{~K} .{ }^{62}$ Although the heating rate used here is relatively low by ReaxFF simulation standards, it is still several orders of magnitude higher than that applied experimentally (ca. $\left.1 \mathrm{~K} \mathrm{~s}^{-1}\right) \cdot{ }^{25,27}$ Thus, the onset temperature for decomposition is expected to be somewhat higher in the current simulations compared to experiments. However, like previous studies of substituent effects with ReaxFF, ${ }^{63}$ the trends between the different molecules and substrates are expected to be accurately represented.

Chemical bonding information was output every 1.0 ps using a bond order cut-off of 0.3 to identify covalent bonds. ${ }^{36}$ It is important to note that the choice of bond order cut-off only affects the post-processing analysis and does not influence the ReaxFF energy or force calculations. ${ }^{62}$ The results shown below represent mean values between three independent simulations with the initial configurations of the phosphate ester molecules shifted by $0.5 \mathrm{~nm}$ in either the $x$ or $y$ direction. ${ }^{37}$ Chemical bonding was thus effectively monitored for 48 molecules for each case, which is sufficient to capture multiple possible reactions. ${ }^{36}$ Videos of the thermal decomposition simulations for some representative systems, TNBP on $\mathrm{Fe}_{3} \mathrm{O}_{4}(001), \alpha-\mathrm{Fe}(110)$, and $a-\mathrm{Fe}_{3} \mathrm{O}_{4}-\mathrm{OH}$, are provided in the Supporting Information.

\section{Results and Discussion}

\section{DFT Validation}

The geometry-optimised structures, adsorption energies, and interatomic distances using ReaxFF and DFT are shown in Fig. 3. It should be noted that, even using DFT, the value of $E_{a d s}$ is highly dependent on the choice of DFT functional. ${ }^{81}$ The ReaxFF parameters used in the current study were developed using the PBE functional. ${ }^{36}$ The DFT calculations used optB86b-vdW, ${ }^{73}$ which gave similar $E_{\text {ads }}$ values to $\mathrm{PBE}^{72}$ for the systems studied here. 
Following geometry optimisation of an intact TNBP molecule adsorbed on $\alpha-\mathrm{Fe}(100), E_{\text {ads }}=$ $2.6 \mathrm{eV}$ with ReaxFF and $E_{a d s}=-1.6 \mathrm{eV}$ with DFT. The DFT adsorption energy is in close agreement $\left(E_{\text {ads }}=-1.9 \mathrm{eV}\right)$ with previous calculations of an intact TCP molecule on $\alpha-\mathrm{Fe}(110)$ using the PBE functional. ${ }^{29}$ The intact TNBP molecule is more strongly adsorbed using ReaxFF compared to DFT, as has been noted in previous comparisons of polar molecules on metal surfaces. ${ }^{82}$ Following, C-O dissociation, $E_{\text {ads }}=-3.8 \mathrm{eV}$ with ReaxFF and $E_{\text {ads }}=-3.6 \mathrm{eV}$ with DFT, while after P-O dissociation, $E_{a d s}=-0.2$ with ReaxFF and $E_{a d s}=-1.4 \mathrm{eV}$ with DFT. These observations indicate that, although the adsorption energy is generally overestimated compared to DFT, the ReaxFF parameterisation ${ }^{36}$ can describe the chemisorption interactions between phosphate esters (both intact and dissociated) and ferrous surfaces. Moreover, the energy differences between the intact and dissociated molecules are in good agreement, as shown in Fig. 4.
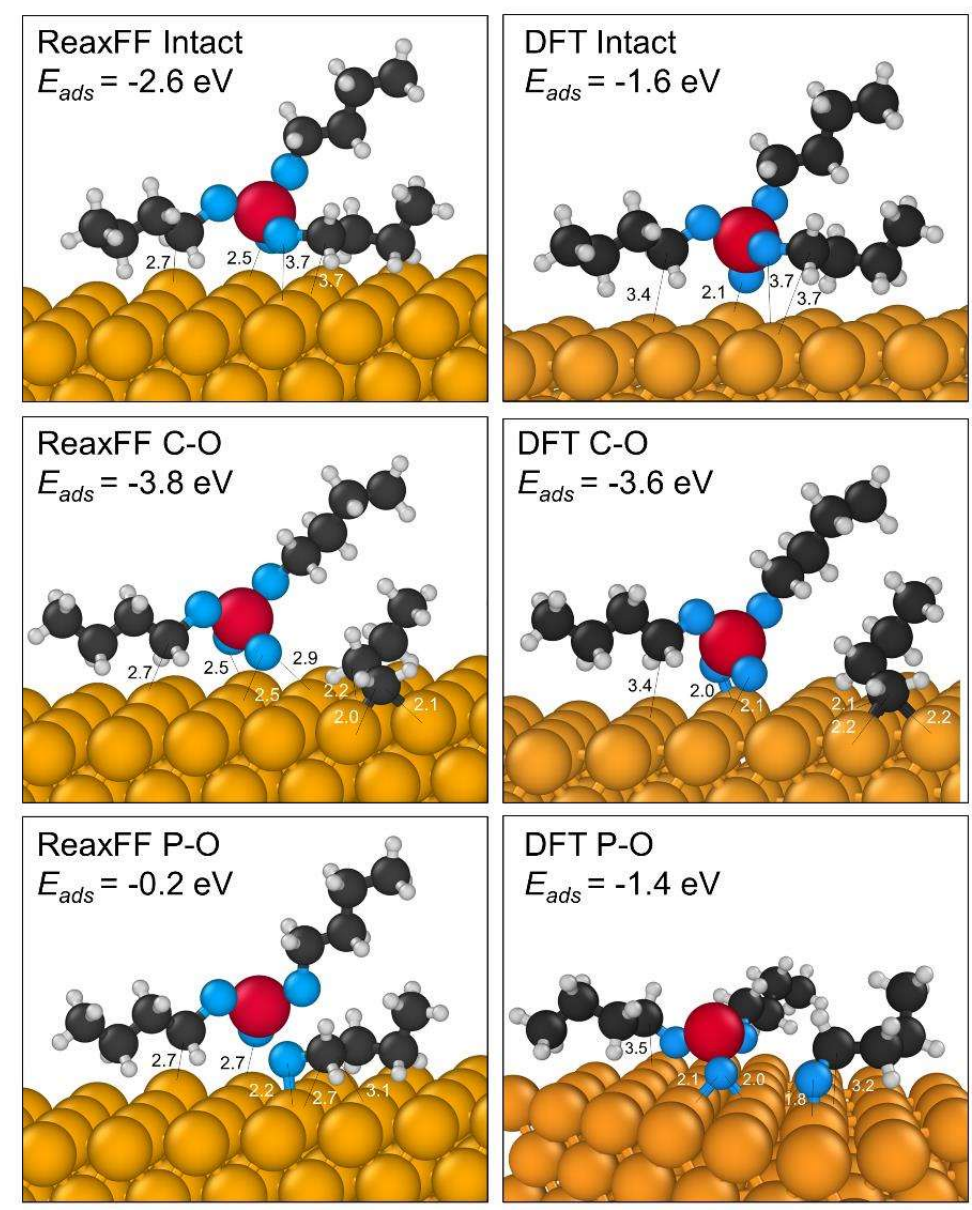

Figure 3. Geometry optimised structures, adsorption energies, and interatomic distances (in A) of an intact and dissociated TNBP molecules on a-Fe(100) using ReaxFF and DFT. Visualised with OVITO,$^{50}$ atom colours are the same as in Fig. 2. 
The initial intact and final dissociated structures following geometry optimisation with DFT (Fig. 3) are quite similar. For the adsorbed intact TNBP molecule, two $\mathrm{O}$ atoms form O-Fe bonds with atoms in the $\alpha-\mathrm{Fe}(100)$ surface. The O-Fe bond distance is $2.5 \AA$ with ReaxFF and $2.1 \AA$ with DFT for the $\mathrm{O}$ atom in the $\mathrm{P}=\mathrm{O}$ bond, while for the $\mathrm{O}$ atom in the closest $\mathrm{P}-\mathrm{O}$ bond it is 3.7 $\AA$ with both ReaxFF and DFT. For the C-O dissociated molecule, the two O-Fe bond distances are both $2.5 \AA$ with ReaxFF while they are $2.0 \AA$ and $2.1 \AA$ with DFT. C-O dissociation forms $n$-butyl radicals which adsorb in a bridge position on the surface, with $\mathrm{C}$-Fe bond distances of $2.0 \AA$ with ReaxFF and $2.2 \AA$ with DFT. The conformation of the $n$-butyl radical and the molecule-surface bond distances are consistent with previous DFT calculations using iron carbide surfaces. ${ }^{83}$ For the P-O dissociated molecule, the O-Fe distance for the $\mathrm{O}$ atom in the closest P-O bond is $2.7 \AA$ for ReaxFF and $2.0 \AA$ for DFT. The $\mathrm{O}-\mathrm{Fe}$ distance for the $\mathrm{O}$ atom in the alkoxy group formed by $\mathrm{P}-\mathrm{O}$ dissociation is $2.2 \AA$ with ReaxFF and $1.8 \AA$ with DFT. In general, the phosphate groups are pulled closer to the surface in DFT, while the alkyl groups are closer with ReaxFF.

Fig. 4 shows the structures and relative energies from the NEB calculations using ReaxFF for C-O cleavage (a) and P-O cleavage (b) within a TNBP molecule on $\alpha-\mathrm{Fe}(100)$. Relative energies for the intact, transition state, and dissociated molecules from single-point DFT calculations (DFT-SP) are shown for comparison. Relative energies for the intact and dissociated structures following geometry optimisation with DFT (DFT-GO) are also shown. DFT-SP provides a direct comparison of the relative energies obtained using the same atom coordinates as ReaxFF, whereas DFT-GO shows relative energies when the atom coordinates are relaxed using DFT. The energies in Fig. 4 are relative to $E_{a d s}$ for an intact TNBP molecule on the $\alpha-\mathrm{Fe}(100)$ surface using the respective methods. For endothermic P$\mathrm{O}$ dissociation, the transition state is product-like so it is omitted for clarity. 
(a)

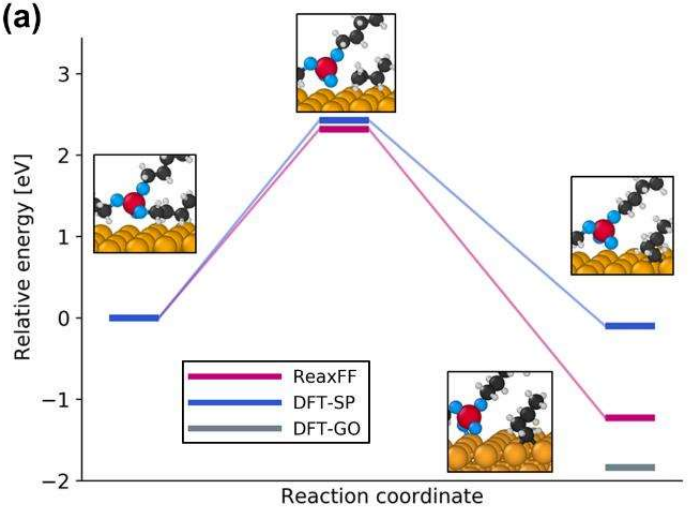

(b)

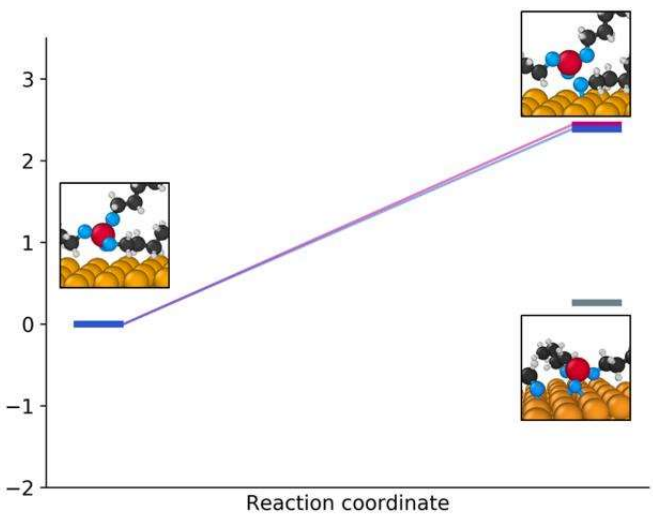

Figure 4. Structures and relative energies from NEB calculations using ReaxFF for $\mathrm{C}-\mathrm{O}$ cleavage (a) and P-O cleavage (b) in TNBP on $\alpha-F e(100)$. DFT relative energies from singlepoint calculations on the ReaxFF NEB structures (DFT-SP) and from geometry optimisation (DFT-GO). Energies are relative to $E_{a d s}$ for the intact molecule using the respective methods.

Fig. 4 shows that, with ReaxFF, C-O dissociation is exothermic with an energy benefit of -1.2 $\mathrm{eV}$ and an activation energy barrier of $2.3 \mathrm{eV}$. Single-point DFT calculations of the initial intact, transition state, and final dissociated structures yield a smaller energy benefit of $-0.3 \mathrm{eV}$, but a similar activation barrier of $2.4 \mathrm{eV}$. When the intact and dissociated structures are both geometry optimised with DFT, the energy benefit is $-2.0 \mathrm{eV}$, which is larger than with ReaxFF. $\mathrm{P}-\mathrm{O}$ cleavage is endothermic with both ReaxFF and single-point DFT calculations, with an similar energy penalty of $2.2 \mathrm{eV}$ and $2.3 \mathrm{eV}$ respectively. P-O dissociation is also endothermic in the geometry optimised DFT calculations, but with a much smaller energy penalty of $0.2 \mathrm{eV}$. The ReaxFF and DFT calculations both suggest that C-O cleavage is the primary thermal decomposition mechanism for alkyl phosphates on ferrous surfaces. The energy changes and barriers for further dissociation reactions in the alkyl phosphate molecules are expected to be similar to the first, as observed in previous DFT calculations of alkyl phosphites on $\alpha-F e(110) .{ }^{31}$

The general agreement between the DFT calculations and the ReaxFF parameterisation ${ }^{36}$ for the stable conformations, relative energies, and energy barriers for the major decomposition processes provides evidence that these reactions will be accurately represented in ReaxFF MD simulations.

\section{Simulations}

Following validation of the ReaxFF parametrisation with DFT, heating MD simulations were performed to study the thermal decomposition of the phosphate esters on ferrous surfaces. The number of bonds between the phosphate ester molecules and the surfaces, as well as 
within the phosphate ester molecules, were monitored as the temperature was linearly increased from 300 to $1000 \mathrm{~K}$ over $1.4 \mathrm{~ns}$ (heating rate of $0.5 \mathrm{~K} \mathrm{ps}^{-1}$ ). Fig. 5 compares the change in the number of bonds formed between the different phosphate ester molecules and the $\mathrm{Fe}_{3} \mathrm{O}_{4}(001)$ surface during heating. The solid lines are rolling-averaged results (taken every $10 \mathrm{ps}$ ) between three independent simulations with different initial positions and the shaded areas represent one standard deviation. The temporal change in the bonding information is consistent between the independent simulations.

Fig. 5 shows that, at room temperature, $<20$ surface-molecule bonds are formed, suggesting that the phosphate esters are mostly physisorbed on $\mathrm{Fe}_{3} \mathrm{O}_{4}(001)$. As the temperature is increased, many more bonds form between the molecules and the surface, indicating that the phosphate esters begin to chemisorb on $\mathrm{Fe}_{3} \mathrm{O}_{4}(001)$. In general, the number of chemisorption interactions increases with increasing temperature and begins to asymptote at high temperature $(>900 \mathrm{~K})$. These observations are consistent with quartz crystal microbalance (QCM) experiments of TCP under UHV conditions which indicated that the adsorbed amount of TCP on Fe a surface saturated at a value that increased with increasing temperature (between 200-500 K). ${ }^{84}$ The simulation results are also in agreement with temperatureprogrammed desorption (TPD) and Auger electron spectroscopy (AES) experiments under UHV conditions which showed that TNBP molecules adsorb strongly on $\mathrm{Fe}_{3} \mathrm{O}_{4}{ }^{27}$

Most of the surface-molecule bonds formed in Fig. 5 originate from the alkyl and aryl groups, more specifically $\mathrm{H}-\mathrm{O}_{\text {surf, }} \mathrm{C}-\mathrm{Fe}$, and $\mathrm{C}-\mathrm{O}_{\text {surf }}$ bonds. This observation suggests that hydroxyl, alkyl/aryl, and alkoxy/aryloxy groups were formed on the surface as the temperature was increased. The formation of these groups has been observed in TPD experiments for both TCP and TNBP on ferrous surfaces under UHV conditions. ${ }^{25,26}$ Chemisorption interactions between atoms in the phosphate group and the surface, for example $\mathrm{O}-\mathrm{Fe}$ and $\mathrm{P}-\mathrm{O}_{\text {surf, }}$ also occur at high temperature $(>600 \mathrm{~K})$. The presence of these bonds confirms iron phosphate formation, as noted from experimental results. ${ }^{25,26}$ The number of molecule-surface chemisorption interactions is quite similar between the different phosphate esters studied. The adsorption of phosphate esters with these substituents has not been studied on ferrous surfaces. However, adsorption experiments of $n$-butyl, $i$-butyl, and $s$-butyl ZDDPs from $n$ hexadecane showed similar adsorbed amounts on iron oxide for the different substituents, ${ }^{49}$ which is consistent with the results in Fig. 5. All of the phosphate esters form a similar number of $\mathrm{H}-\mathrm{O}_{\text {surf }}$ bonds, while aryl phosphates form more $\mathrm{C}$-Fe bonds than alkyl phosphates. This observation can be rationalised through the higher total number of $C$ atoms for the aryl phosphate systems (336) compared to the alkyl phosphates (192). Negligible P-Fe bond formation occurs during any of the simulations, confirming that iron phosphate, ${ }^{11-13}$ rather than iron phosphide ${ }^{10}$ is formed on ferrous surfaces from phosphate ester molecules. 
Temperature $[\mathrm{K}]$

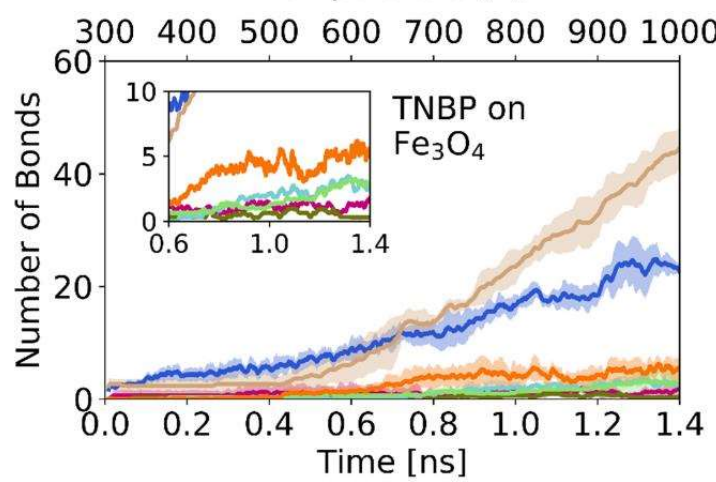

Temperature $[\mathrm{K}]$

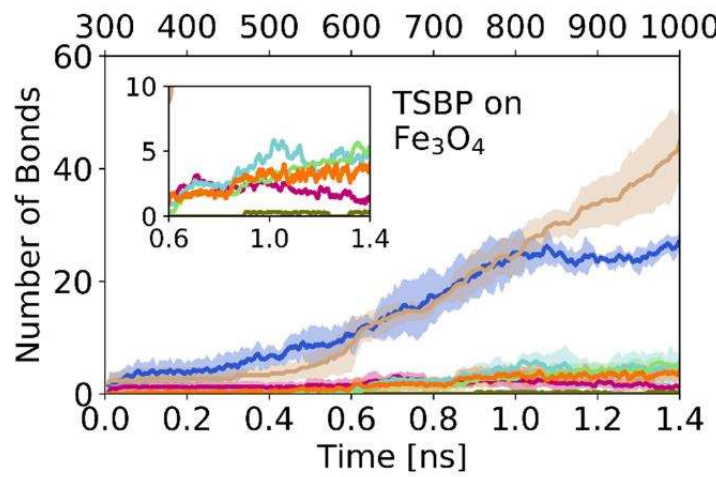

Temperature $[\mathrm{K}]$

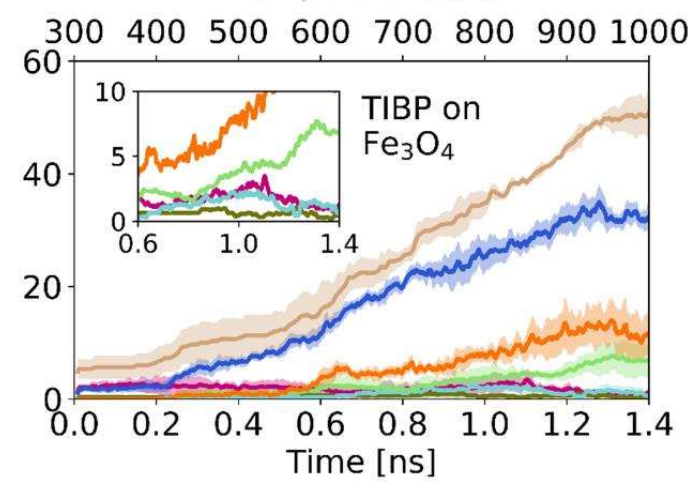

Temperature $[\mathrm{K}]$

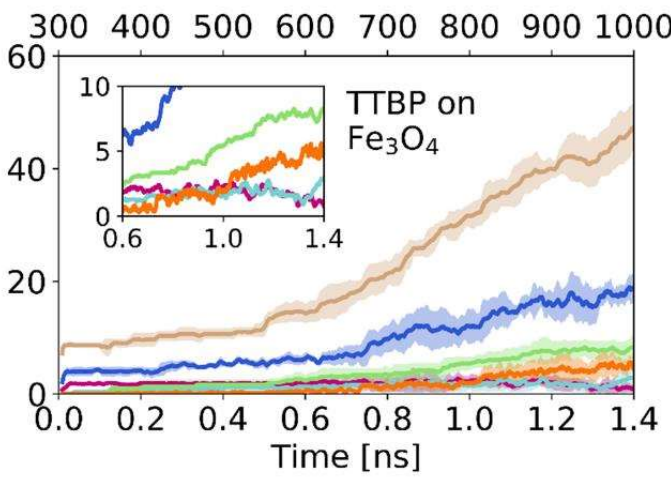

Temperature $[\mathrm{K}]$
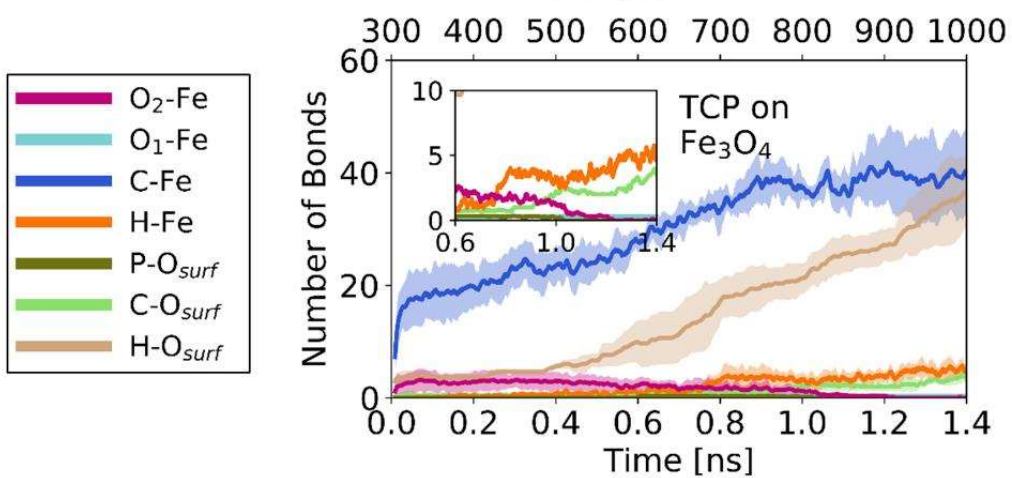

Figure 5. Number of covalent bonds formed between TNBP, TIBP, TSBP, TTBP, TCP and the $\mathrm{Fe}_{3} \mathrm{O}_{4}(001)$ surface. Each line represents the mean of three independent simulations and shaded areas represent one standard deviation. Insets show magnifications of the mean results in the second half of the simulation for the less commonly observed bonds.

In addition to the molecule-surface bond formation, it is also interesting to study bond breaking within the phosphate ester molecules, since this initiates polyphosphate tribofilm formation. ${ }^{16}$ Fig. 6 compares the percentage of intact $\mathrm{C}-\mathrm{H}, \mathrm{C}-\mathrm{O}$, and $\mathrm{P}-\mathrm{O}$ bonds remaining within the 
phosphate ester molecules as the temperature is linearly increased from $300-1000 \mathrm{~K}$ on $\mathrm{Fe}_{3} \mathrm{O}_{4}(001)$. The percentages are relative to the intact molecules after energy minimisation and equilibration. The total number of $\mathrm{C}-\mathrm{H}, \mathrm{C}-\mathrm{O}, \mathrm{P}-\mathrm{O}, \mathrm{P}=\mathrm{O}$, and $\mathrm{C}-\mathrm{C}$ bonds remaining within the molecules during heating on $\mathrm{Fe}_{3} \mathrm{O}_{4}(001)$ is shown in the Supporting Information (Fig. S1). Negligible $\mathrm{C}-\mathrm{C}$ or $\mathrm{P}=\mathrm{O}$ bond scission occurs for any of the phosphate esters on $\mathrm{Fe}_{3} \mathrm{O}_{4}(001)$.

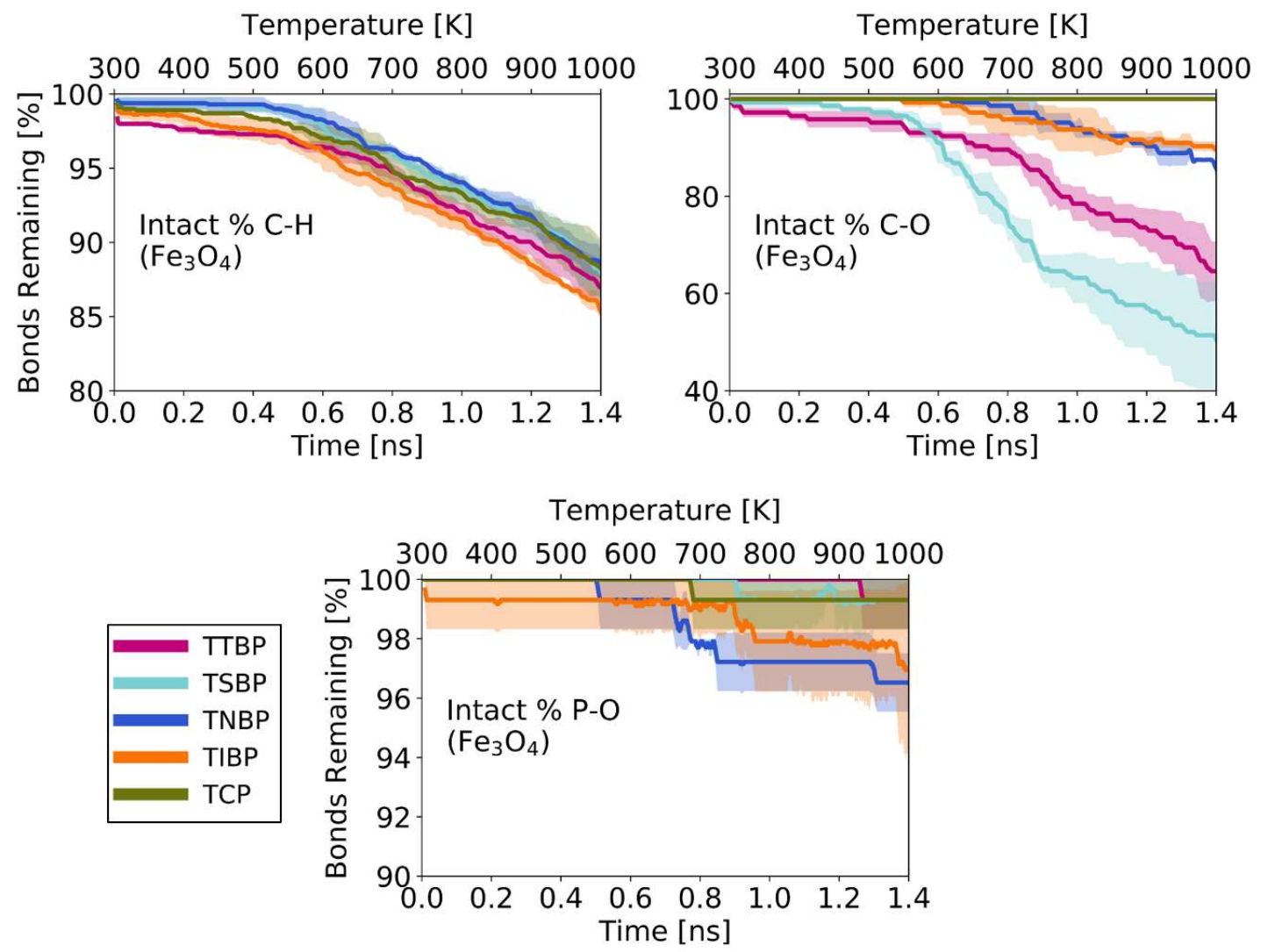

Figure 6. Percentage of intact $\mathrm{C}-\mathrm{O}, \mathrm{C}-\mathrm{H}$, and $\mathrm{P}-\mathrm{O}$ bonds for TNBP, TIBP, TSBP, TTBP, and TCP on the $\mathrm{Fe}_{3} \mathrm{O}_{4}(001)$ surface. The percentages are relative to the intact molecules after energy minimisation. Solid lines are means of three independent simulations, shaded areas represent one standard deviation.

Fig. 6 shows that $\mathrm{C}-\mathrm{H}$ cleavage occurs for all of the phosphate esters, even at low temperatures. Dehydrogenation of the alkyl and aryl groups leads to the $\mathrm{H}-\mathrm{O}_{\text {surf }}$ bond formation (hydroxylation) observed in Fig. 5. Iron oxides are known to be effective alkane dehydrogenation catalysts, ${ }^{85}$ and dehydrogenation has been observed experimentally for TNBP on $\mathrm{Fe}_{3} \mathrm{O}_{4}$ at low temperature $(300 \mathrm{~K})$ using TPD. ${ }^{27}$ Fig. 6 shows that both the onset temperatures for $\mathrm{C}-\mathrm{H}$ cleavage and the rates of dehydrogenation are similar for all of the phosphate esters studied on $\mathrm{Fe}_{3} \mathrm{O}_{4}(001)$. Dehydrogenation begins at relatively low 
temperature $(\approx 400 \mathrm{~K})$, and $10-15 \%$ of the $\mathrm{C}-\mathrm{H}$ bonds are broken by the end of the heating simulations $(1000 \mathrm{~K})$ for all of the phosphate esters studied.

Clear differences between the various phosphate esters are observed in terms of the onset temperature and rate of $\mathrm{C}-\mathrm{O}$ cleavage in Fig 6. The tertiary phosphate (TTBP) has the lowest onset temperature ( $<350 \mathrm{~K}$ ) for $\mathrm{C}-\mathrm{O}$ cleavage on $\mathrm{Fe}_{3} \mathrm{O}_{4}(001)$, and approximately $40 \%$ of the $\mathrm{C}-\mathrm{O}$ bonds are broken by the end of the simulation $(1000 \mathrm{~K})$. For the secondary phosphate (TSBP), C-O cleavage begins at higher temperature ( $500 \mathrm{~K})$ compared to TTBP; however, at high temperature (>600 K), TSBP has a higher rate of C-O cleavage, and more of the C-O bonds $(50 \%)$ are broken by the end of the simulation. For both primary alkyl phosphates (TNBP and TIBP), C-O cleavage begins at approximately $600 \mathrm{~K}$, and only $10 \%$ of the C-O bonds are broken by the end of the simulation. Negligible $\mathrm{C}-\mathrm{O}$ cleavage occurs during the TCP simulations on $\mathrm{Fe}_{3} \mathrm{O}_{4}(001)$, even at very high temperature $(1000 \mathrm{~K})$. The differences between the different substituents can be rationalised based on the relative stability of the radicals formed by $\mathrm{C}-\mathrm{O}$ dissociation.

In the thermal decomposition simulations, $\mathrm{P}-\mathrm{O}$ cleavage is much less prevalent than $\mathrm{C}-\mathrm{H}$ and C-O cleavage for all of the alkyl phosphate esters studied, with $<5 \%$ of the bonds broken at the end of the simulation. The P-O cleavage reactions which do occur proceed via nucleophilic substitution by surface $O$ atoms. In general, primary alkyl phosphates (TNBP and TIBP) show more P-O cleavage compared to secondary alkyl (TSBP), tertiary alkyl (TTBP), and aryl (TCP) phosphates. This decrease in $\mathrm{P}-\mathrm{O}$ cleavage is probably due to increased steric hindrance, which reduces the probability of nucleophilic attack. ${ }^{86}$ Although nucleophiles are present in the simulations in the form of $\mathrm{O}$ atoms in the phosphate ester molecules and the surface, these are relatively immobile on MD simulation timescales.

Overall, Fig. 6 suggests that alkyl phosphate thermal decomposition proceeds mainly via C-O and $\mathrm{C}-\mathrm{H}$ cleavage on $\mathrm{Fe}_{3} \mathrm{O}_{4}(001)$, with some $\mathrm{P}-\mathrm{O}$ cleavage occurring at high temperatures. For aryl phosphates, $\mathrm{P}-\mathrm{O}$ and $\mathrm{C}-\mathrm{H}$ cleavage are the only decomposition mechanisms that occur on $\mathrm{Fe}_{3} \mathrm{O}_{4}(001)$.

Fig. 7 shows snapshots of representative phosphate ester molecules (TNBP) which undergo $\mathrm{C}-\mathrm{O}$ and $\mathrm{P}-\mathrm{O}$ bond cleavage on $\mathrm{Fe}_{3} \mathrm{O}_{4}(001)$ during the heating simulations. During $\mathrm{C}-\mathrm{O}$ dissociation, the primary $\alpha-C$ radical is stabilised by bond formation with a surface Fe atom and, in this case, the phosphate radical forms a new $\mathrm{O}-\mathrm{H}$ bond from a surface hydroxyl group formed from a separate dehydrogenation reaction. During $\mathrm{P}-\mathrm{O}$ cleavage, an $\mathrm{O}_{\text {surf }}$ atom forms a bond with a $\mathrm{P}$ atom in the TNBP molecule, as expected for nucleophilic substitution. The resultant alkoxy group bonds to a surface $\mathrm{Fe}$ atom. 


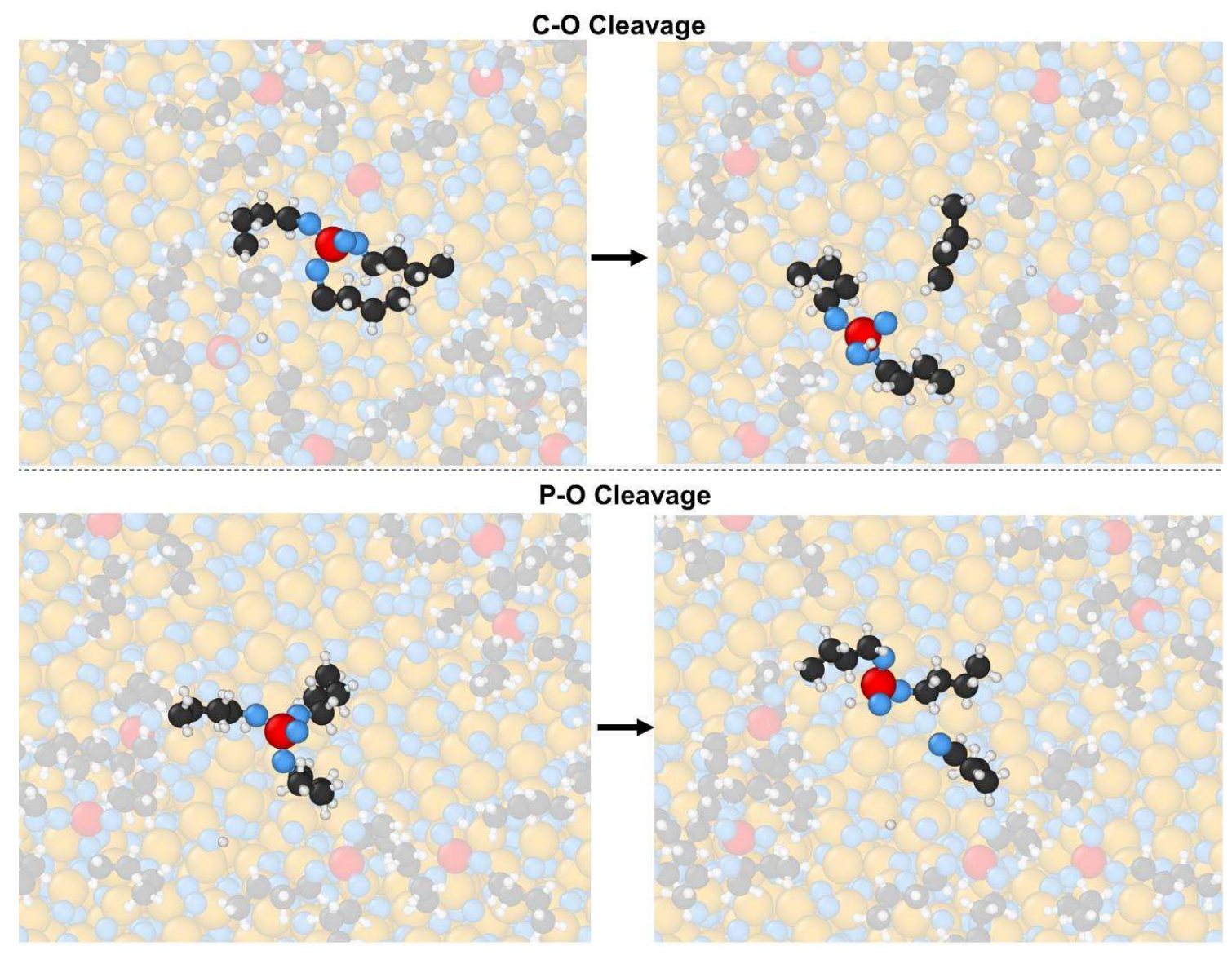

Figure 7. Snapshots showing a representative phosphate ester molecule (TNBP) on the $\mathrm{Fe}_{3} \mathrm{O}_{4}(001)$ surface before (left) and after (right) $\mathrm{C}-\mathrm{O}$ bond cleavage (top) and P-O bond cleavage (bottom). All of the atoms are translucent except those in a representative TNBP molecule in which dissociation occurs during the heating simulations. Visualised with OVITO, ${ }^{50}$ atom colours are the same as in Fig. 2.

In the absence of metal or metal oxide surfaces, alkyl phosphates typically thermally decompose above around $530 \mathrm{~K}$, while aryl phosphates remain stable up to approximately $640 \mathrm{~K} .{ }^{20}$ TPD and AES experiments have shown that TNBP molecules begin to dissociate at room temperature on iron oxide surfaces ${ }^{27}$ and XPS experiments have indicated that TCP decomposes at higher temperature (400-500 K) on steel surfaces. ${ }^{87}$ The onset temperatures for thermal decomposition on $\mathrm{Fe}_{3} \mathrm{O}_{4}(001)$ in Fig. 6 are thus approximately $100 \mathrm{~K}$ higher in the ReaxFF MD simulations than observed experimentally. This is probably a consequence of the much higher heating rate in the simulations $\left(0.5 \mathrm{~K} \mathrm{ps}^{-1}\right)$ compared to the experiments $\left(1 \mathrm{~K} \mathrm{~s}^{-}\right.$ $\left.{ }^{1}\right),{ }^{25,27}$ which has been shown previously to lead to overestimated decomposition temperatures. ${ }^{62}$ While the onset temperature for decomposition is overestimated in the 
simulations, the relative trends between the different phosphate esters are in remarkable agreement with experimental observations for both phosphate esters and ZDDPs. In particular, aryl phosphates show a much higher onset temperature for decomposition compared to alkyl phosphates. ${ }^{20}$ Moreover, the onset temperature for $\mathrm{C}-\mathrm{O}$ cleavage on $\mathrm{Fe}_{3} \mathrm{O}_{4}(001)$ increases in the order: TTBP (tertiary alkyl) < TSBP (secondary alkyl) < TNBP (primary linear alkyl) $\approx$ TIBP (primary branched alkyl) < TCP (aryl). This order is mostly consistent with the thermal stability of ZDDPs with similar substituents in base oil solution. ${ }^{86}$ In the glassware experiments, without ferrous surfaces, primary branched ZDDPs were found to be less thermally stable compared to primary linear ones. This was attributed to slower PO cleavage in the former due to increased steric hindrance. ${ }^{86}$ However, in the current ReaxFF MD study, the phosphate esters with these substituents showed similar decomposition rates on $\mathrm{Fe}_{3} \mathrm{O}_{4}(001)$. This is because thermal decomposition proceeds mainly through $\mathrm{C}-\mathrm{O}$ cleavage, which will not be retarded by steric effects.

We also investigated the thermal decomposition of phosphate esters on non-passivated $\alpha$ $\mathrm{Fe}(110)$ and hydroxylated $a-\mathrm{Fe}_{3} \mathrm{O}_{4}-\mathrm{OH}$ surfaces. Fig. 8 compares the change in the number of bonds formed between primary linear (TNBP) and secondary (TSBP) alkyl phosphate ester molecules on $\alpha-\mathrm{Fe}(110)$ and $a-\mathrm{Fe}_{3} \mathrm{O}_{4}-\mathrm{OH}$. Many more surface-molecule bonds are formed on a-Fe(110) compared to $\mathrm{Fe}_{3} \mathrm{O}_{4}(001)$ (Fig. 5). In particular, C-Fe, $\mathrm{O}_{1}-\mathrm{Fe}$, and $\mathrm{O}_{2}-\mathrm{Fe}$ bonding begins at lower temperature and around twice as many bonds are formed by the end of the heating simulation. Previous AIMD simulations of tri(methyl)phosphate also suggested that $\mathrm{O}_{1}-\mathrm{Fe}$ and $\mathrm{O}_{2}-\mathrm{Fe}$ bond formation readily occurs on $\alpha-\mathrm{Fe}(110) .{ }^{30}$ This observation suggests that, if nascent $\alpha-F e$ is exposed during rubbing, ${ }^{46}$ it is quickly passivated by phosphate ester molecules. ${ }^{88}$ Since so many strong binding chemisorption interactions occur on $\alpha-\mathrm{Fe}(110)$, fewer species are released into the gas phase compared to the other surfaces (see Supporting Information videos).

Conversely, Fig. 8 indicates that very few chemisorption interactions occur between the a$\mathrm{Fe}_{3} \mathrm{O}_{4}-\mathrm{OH}$ surface and the alkyl phosphate esters, which is consistent with previous ReaxFF MD simulations for TCP. ${ }^{36,37}$ Similarly, experiments have shown that TCP physisorbs on inert gold surfaces and desorbs above $500 \mathrm{~K}$, rather than decomposing and forming a film as it does on more reactive ferrous surfaces. ${ }^{87}$ Indeed, desorption of intact molecules is observed on a- $\mathrm{Fe}_{3} \mathrm{O}_{4}-\mathrm{OH}$, but not the other ferrous surfaces studied here (see Supporting Information videos). This suggests that phosphate esters will only physisorb on regions of the surface that are hydroxylated and desorb at high temperature, rather than decomposing to initiate film formation. 
Temperature $[\mathrm{K}]$

3004005006007008009001000

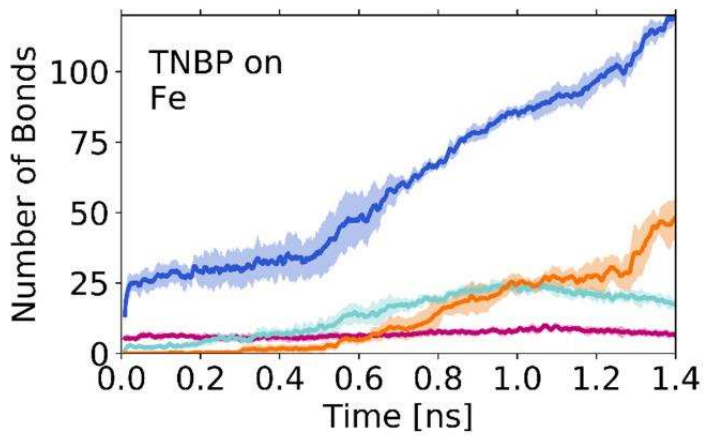

Temperature $[\mathrm{K}]$

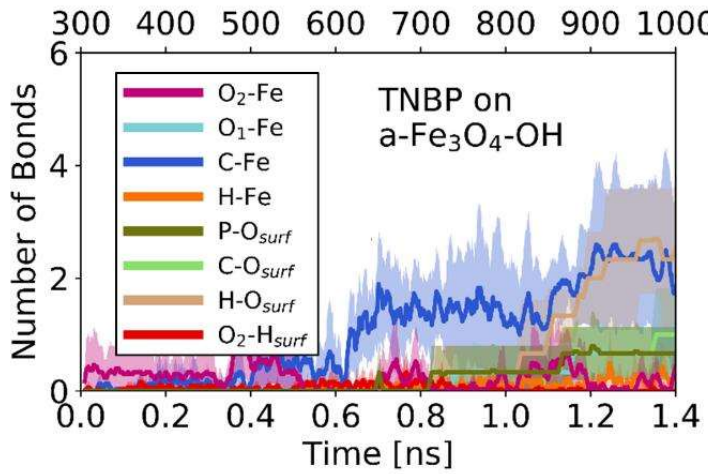

Temperature $[\mathrm{K}]$

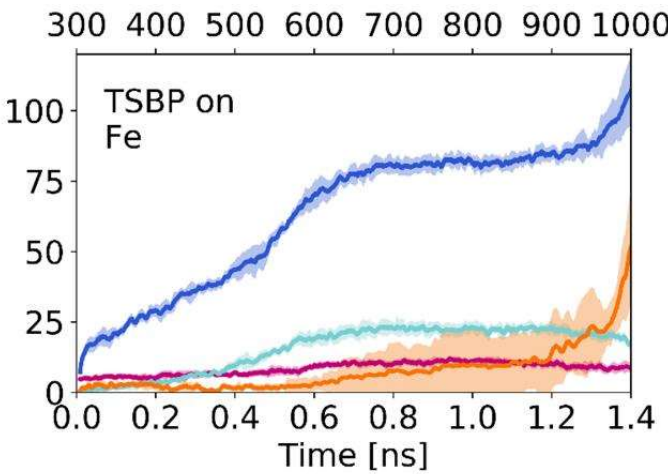

Temperature $[\mathrm{K}]$

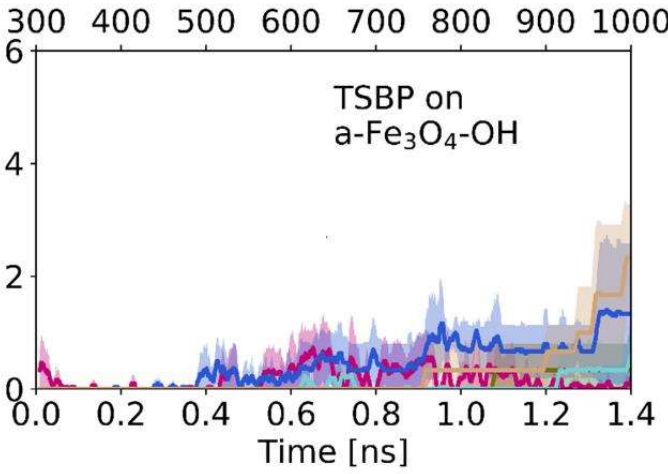

Figure 8. Number of covalent bonds formed between TNBP/TSBP molecules and the $\alpha-$ $\mathrm{Fe}(110) / a-\mathrm{Fe}_{3} \mathrm{O}_{4}-\mathrm{OH}$ surfaces. Solid lines are means of three independent simulations, shaded areas represent one standard deviation.

Fig. 9 compares the change in the percentage of intact $\mathrm{C}-\mathrm{O}, \mathrm{C}-\mathrm{H}$, and $\mathrm{P}-\mathrm{O}$ bonds remaining for the different ferrous surfaces during the heating simulations. The total number of $\mathrm{C}-\mathrm{H}, \mathrm{C}-$ $\mathrm{O}, \mathrm{P}-\mathrm{O}, \mathrm{P}=\mathrm{O}$, and $\mathrm{C}-\mathrm{C}$ bonds remaining within the molecules during heating on the different surfaces is shown in the Supporting Information (Fig. S2). For both primary linear (TNBP) and secondary (TSBP) alkyl phosphates, $\mathrm{C}-\mathrm{H}$ cleavage occurs at room temperature on $\mathrm{Fe}_{3} \mathrm{O}_{4}(001)$, at higher temperature $(>500 \mathrm{~K})$ on $\alpha-\mathrm{Fe}(110)$, and only at very high temperature $(>700 \mathrm{~K})$ on $a-\mathrm{Fe}_{3} \mathrm{O}_{4}-\mathrm{OH}$. This observation can be attributed to the fact that $\mathrm{H}-\mathrm{O}_{\text {surf }}$ bond formation facilitates the formation of stable hydroxyl groups while C-Fe bonding stabilises the resultant alkene, as shown in Fig. 5. Although $\mathrm{H}$-Fe bonding does occur on $\mathrm{\alpha}-\mathrm{Fe}(110)$ surfaces in Fig. 8, this requires higher temperatures, suggesting a larger activation energy. Since a$\mathrm{Fe}_{3} \mathrm{O}_{4}-\mathrm{OH}$ already contains surface hydroxyls, it is unsurprising that $\mathrm{C}-\mathrm{H}$ dissociation requires very high temperatures. $\mathrm{C}-\mathrm{O}$ cleavage begins at lower temperatures and more $\mathrm{C}-\mathrm{O}$ bonds dissociate on $\alpha-\mathrm{Fe}(110)$ compared to $\mathrm{Fe}_{3} \mathrm{O}_{4}(001)$ and particularly a- $\mathrm{Fe}_{3} \mathrm{O}_{4}-\mathrm{OH}$. Surface $\mathrm{Fe}$ 
atoms are more accessible on $\alpha-\mathrm{Fe}(110)$ to promote $\beta-\mathrm{H}$ elimination than on $\mathrm{Fe}_{3} \mathrm{O}_{4}(001)$ and a- $\mathrm{Fe}_{3} \mathrm{O}_{4}-\mathrm{OH}$. Fig. 8 also shows that, on $\alpha-\mathrm{Fe}(110), \mathrm{C}-\mathrm{O}$ cleavage occurs at lower temperature ( $350 \mathrm{~K})$ for TSBP compared to TNBP $(400 \mathrm{~K})$; however, the difference in onset temperature between the molecules is smaller than on $\mathrm{Fe}_{3} \mathrm{O}_{4}(001)$.

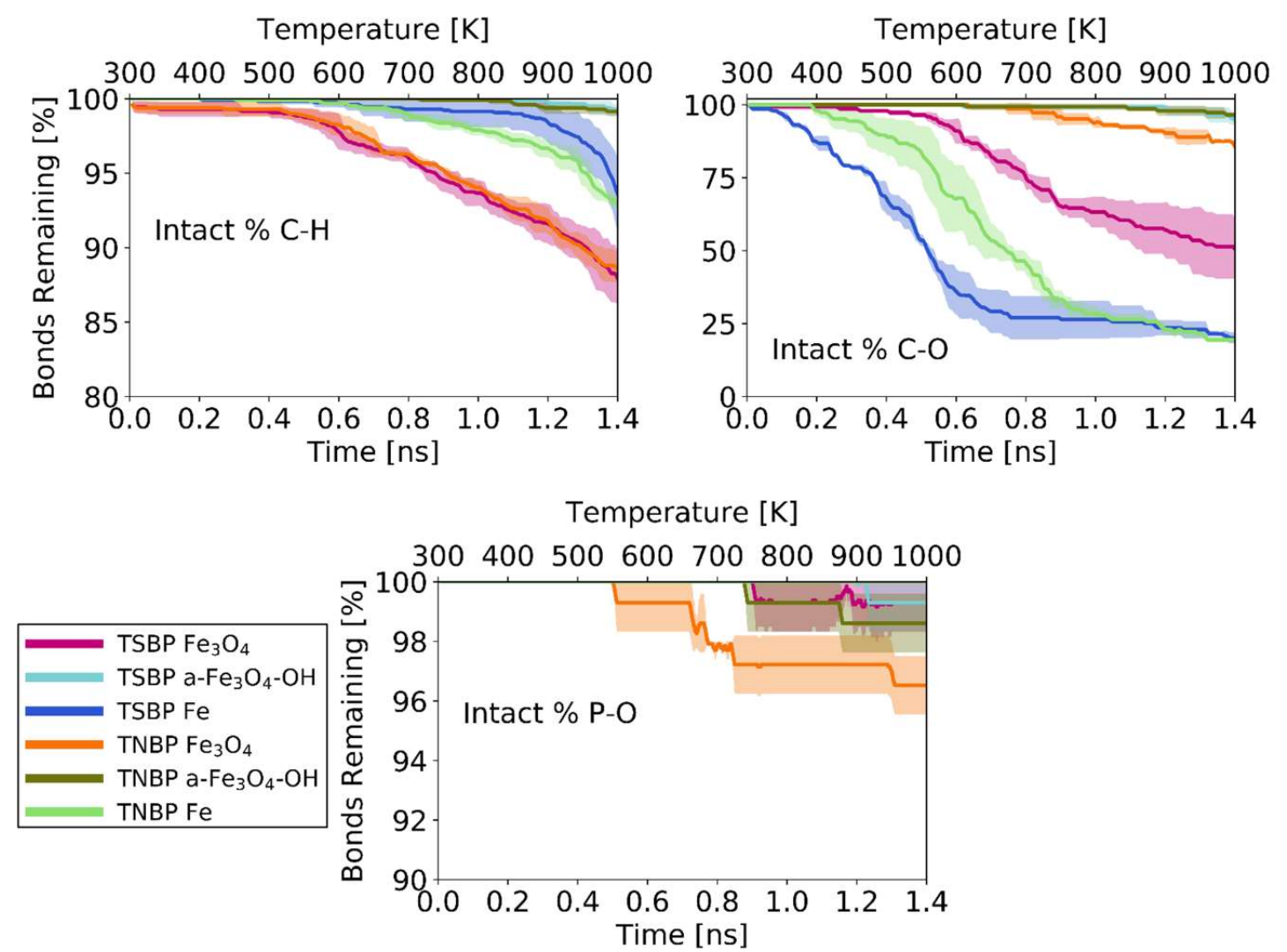

Figure 9. Percentage of intact $\mathrm{C}-\mathrm{O}, \mathrm{C}-\mathrm{H}$, and $\mathrm{P}-\mathrm{O}$ bonds for TNBP and TSBP on the $\alpha-$ $\mathrm{Fe}(110), \mathrm{Fe}_{3} \mathrm{O}_{4}(001)$, and $a-\mathrm{Fe}_{3} \mathrm{O}_{4}-\mathrm{OH}$ surfaces. The percentages are relative to the intact molecules after energy minimisation. Solid lines are means of three independent simulations, shaded areas represent one standard deviation.

In Fig. 9, some P-O cleavage occurs on $\mathrm{Fe}_{3} \mathrm{O}_{4}(001)$ and to a lesser extent a- $\mathrm{Fe}_{3} \mathrm{O}_{4}-\mathrm{OH}$; however, no P-O bonds are broken on $\alpha-\mathrm{Fe}(110)$. This observation confirms that $\mathrm{P}-\mathrm{O}$ cleavage requires proximal nucleophiles $\left(\mathrm{O}_{\text {surf }}\right.$ atoms) for the phosphate esters studied here. Since $\mathrm{C}$ O dissociation does not occur for aryl phosphates such as TCP (Fig. 6), accelerated P-O scission via nucleophilic substitution can explain their higher reactivity experimentally on iron oxide compared to iron surfaces. ${ }^{15}$ From these simulations, the opposite trend (higher reactivity on iron compared to iron oxide) is expected for alkyl phosphates that undergo C-O dissociation, although this has not yet been experimentally confirmed. 
Compared to the other surfaces, only a very small number of bonds are broken within TNBP and TSBP on a- $\mathrm{Fe}_{3} \mathrm{O}_{4}-\mathrm{OH}$. This observation suggests that phosphate esters form polyphosphate films much more slowly on regions of the surface that are hydroxylated. Indeed, tribology experiments have suggested that TCP tribofilm formation is hindered by the presence of water, ${ }^{19}$ which likely results in surface hydroxylation. ${ }^{79}$ Moreover, TCP is known to be an ineffective antiwear additive on inert oxide films such as aluminium, nickel, and chromium oxide. $^{15}$

For all of the alkyl phosphates and surfaces studied, the primary thermal decomposition mechanism is $\mathrm{C}-\mathrm{O}$ bond cleavage on $\mathrm{Fe}_{3} \mathrm{O}_{4}(001)$ (Fig. 6). This observation is in agreement with experiments for TNBP on $\alpha$-Fe surfaces by Sung and Gellman..$^{25,26}$ The current results do not support the conclusions of Gao et al., ${ }^{27}$ who suggested that $\mathrm{P}-\mathrm{O}$ cleavage was the primary decomposition mechanism for TNBP on $\mathrm{Fe}_{3} \mathrm{O}_{4}$. This postulation was based upon the fact that mostly 1-butanol, rather than 1-butene, was detected during heating in their TPD experiments. ${ }^{27}$ In the simulations on $\mathrm{Fe}_{3} \mathrm{O}_{4}(001)$, both $\mathrm{C}-\mathrm{Fe}$ and $\mathrm{C}-\mathrm{O}_{\text {surf }}$ bonds are formed as the temperature is increased (see Fig. 5), suggesting that both alkyl and alkoxy groups are deposited on the surface following $\mathrm{C}-\mathrm{O}$ cleavage. Furthermore, both gas phase alkene and alcohol molecules are released for all of the alkyl phosphates on $\mathrm{Fe}_{3} \mathrm{O}_{4}(001)$ (see Supporting Information videos). These observations suggest that some of the alkene molecules formed during $\mathrm{C}-\mathrm{O}$ cleavage are oxidised ${ }^{89}$ and hydrogenated before being released into the gas phase as alcohols. The $\mathrm{H}_{\text {surf }}$ atoms required for this process are formed by separate dehydrogenation reactions of the phosphate ester alkyl chains. ${ }^{85}$ Therefore, the lack of gas phase alkenes detected by Gao et al. ${ }^{27}$ in their TPD experiments on $\mathrm{Fe}_{3} \mathrm{O}_{4}$ does not preclude $\mathrm{C}-\mathrm{O}$ cleavage as the primary thermal decomposition mechanism of TNBP (and other alkyl phosphates) on $\mathrm{Fe}_{3} \mathrm{O}_{4}$. Conversely, Sung and Gellman. ${ }^{25,26}$ did observe gas phase alkenes for TNBP on $\alpha-\mathrm{Fe}$, since fewer $\mathrm{O}_{\text {surf }}$ atoms were available for oxidation to form alcohols. For the more thermally stable aryl phosphates (TCP), $\mathrm{P}-\mathrm{O}$ and $\mathrm{C}-\mathrm{H}$ cleavage are the only decomposition mechanism observed in the simulations, since $\mathrm{C}-\mathrm{O}$ scission is disfavoured due to the low stability of benzyl radicals. The surface-adsorbed aryloxy groups formed through P$\mathrm{O}$ cleavage cannot undergo $\beta$ - $\mathrm{H}$ elimination and are thus non-volatile. These observations are in agreement with the conclusions of Sung and Gellman. ${ }^{25,26}$

Decomposition of antiwear additives is crucial to initiate film formation and ultimately wear mitigation. It has recently been shown that the initial removal of alkyl groups is the rate determining step for ZDDP tribofilm formation. ${ }^{90}$ Several researchers have attempted to link the thermal stability of antiwear additives with their tribological performance. For example, it has been suggested that pure aryl phosphates (with higher thermal stability ${ }^{20}$ ) yield improved antiwear performance compared to alkyl phosphates; ${ }^{22}$ however, the opposite trend has been 
reported when they were dissolved in a base oil. ${ }^{23,24}$ Rowe and Dickert ${ }^{91}$ suggested that more thermally stable primary ZDDPs exhibited better antiwear performance compared to secondary ZDDPs at high temperature $(420 \mathrm{~K})$. However, Fujita et al. ${ }^{92}$ showed that ZDDPs with secondary alkyl groups form tribofilms more quickly compared to those with primary alkyl groups at moderate temperature $(370 \mathrm{~K})$. Furthermore, Topolovec-Miklozic et al. ${ }^{93}$ showed that this faster tribofilm formation for secondary ZDDPs could lead to improved antiwear performance compared to primary ZDDPs at moderate temperature ( $370 \mathrm{~K})$.

Most commercial lubricants contain a mixture of primary and secondary ZDDPs ${ }^{94}$ to provide a 'graded response' over a wide range of conditions. ${ }^{95}$ Moreover, lubricants for spark ignition (gasoline) engines generally contain a higher proportion of secondary ZDDPs compared to those for compression ignition (diesel) engines, to provide tribofilms of sufficient thickness at their lower operating temperature. ${ }^{3}$ Molecular simulations could soon provide a molecularlevel understanding of additive decomposition and tribofilm formation to facilitate the rational design of new antiwear additives with optimised performance under the relevant operating conditions.

It should also be noted that, for ZDDP, the films formed by thermal decomposition are significantly different from the tribofilms formed inside rubbing contacts. ${ }^{96}$ It is not yet clear whether this is due to a different formation mechanism or a subsequent stress-induced transformation. ${ }^{97}$ More generally, applied stresses can lead to rather different reaction pathways and products compared to those obtained thermally. ${ }^{98}$ Thus, an important progression in future studies might be to investigate the effect of applied stresses on the decomposition of phosphate esters on ferrous surfaces.

Now that this ReaxFF parameterisation ${ }^{36}$ has been validated for thermal decomposition of a wide range of phosphate esters on different ferrous surfaces, it can be used to study the effect of applied stress on molecular decomposition and tribofilm formation in nonequilibrium molecular dynamics (NEMD) simulations. ${ }^{28}$ Indeed, NEMD simulations have recently been performed for a wide range of other systems using ReaxFF. ${ }^{99-101}$ It will also be useful to validate the results of the ReaxFF NEMD simulations against AIMD simulations. For example, Loehlé and Righi showed that the internal energy of phosphite esters with butyl chains increased more with pressure than those with methyl chains when confined between $\alpha-$ $\mathrm{Fe}(110)$ surfaces, leading to a higher decomposition rate. ${ }^{32}$ AIMD simulations of tri(methyl)phosphate molecules confined between sliding a-Fe surfaces suggested that the applied stress resulted in dissociation of the $\mathrm{P}=\mathrm{O}$ bond, which was suggested to be the initial step of tribofilm formation on $\alpha-F e$ surfaces. ${ }^{30}$ Negligible $P=O$ bond cleavage was observed in 
the thermal decomposition ReaxFF MD simulations performed here (Fig. S1 and S2), so it will be interesting to investigate whether this occurs in NEMD simulations using ReaxFF.

\section{Conclusions}

The thermal decomposition of phosphate esters on ferrous surfaces has been studied using MD simulations with ReaxFF. The ReaxFF parameterisation was validated against DFT calculations for adsorption and dissociation of representative surface-molecule combinations. Following geometry optimisation, there was reasonable agreement between ReaxFF and DFT for the adsorption energy of intact and dissociated TNBP molecules on $\alpha-\mathrm{Fe}(110)$. The relative energy of the intact, transition state, and dissociated structures obtained from NEB calculations using ReaxFF showed close agreement with single-point DFT calculations. With both ReaxFF and DFT, C-O cleavage was exothermic with a relatively small activation energy, while P-O cleavage was endothermic.

The thermal decomposition of five phosphate esters with different substituents was then compared on three ferrous surfaces using ReaxFF MD simulations. In the MD simulations, the temperature was linearly increased from $300-1000 \mathrm{~K}$ over $1.4 \mathrm{~ns}$. For alkyl phosphates on $\mathrm{Fe}_{3} \mathrm{O}_{4}(001)$, thermal decomposition proceeds mostly through $\mathrm{C}$-O cleavage (to form surfaceadsorbed alkyl groups) and $\mathrm{C}-\mathrm{H}$ cleavage (to form surface hydroxyls). Some P-O cleavage also occurs through nucleophilic substitution at high temperature on $\mathrm{Fe}_{3} \mathrm{O}_{4}(001)$, but this is a much rarer process. As expected based on previously reported experiments, alkyl phosphates decompose at lower temperatures compared to aryl phosphates, which only decompose through $\mathrm{P}-\mathrm{O}$ and $\mathrm{C}-\mathrm{H}$ dissociation. The onset temperature for $\mathrm{C}-\mathrm{O}$ cleavage on $\mathrm{Fe}_{3} \mathrm{O}_{4}(001)$ increases in the order; tertiary alkyl (TTBP) < secondary alkyl (TSBP) < primary linear alkyl $(\mathrm{TNBP}) \approx$ primary branched alkyl $(\mathrm{TSBP})<$ aryl $(\mathrm{TCP})$. This order can be rationalised through the relative stability of the radicals formed through $\mathrm{C}-\mathrm{O}$ dissociation. The order is also mostly consistent with experiments for the thermal stability of ZDDPs with similar substituents in base oil solution.

On $\mathrm{Fe}_{3} \mathrm{O}_{4}(001)$ and $\alpha-\mathrm{Fe}(110)$ surfaces, chemisorption interactions between the molecules and the surface occur even at room temperature and the number of bonds increases at higher temperature. On these substrates, the early stages of iron phosphate formation are observed at high temperature, as expected from experiments. Conversely, on a- $\mathrm{Fe}_{3} \mathrm{O}_{4}-\mathrm{OH}$, most of the molecules are only physisorbed, even at high temperature. Very few chemisorption interactions or dissociation events occur on a- $\mathrm{Fe}_{3} \mathrm{O}_{4}-\mathrm{OH}$ compared to the other surfaces, and desorption of intact molecules is observed at high temperature. These observations could account for the poor antiwear performance of phosphate esters on inert surfaces noted in tribology experiments. The rate of thermal decomposition is higher on $\mathrm{Fe}_{3} \mathrm{O}_{4}(001)$, and 
particularly $\alpha-\mathrm{Fe}(110)$ compared to $a-\mathrm{Fe}_{3} \mathrm{O}_{4}-\mathrm{OH}$. This decrease in rate suggests that water passivates ferrous surfaces and inhibits thermal decomposition and thus film formation of phosphate esters. The observed differences between the various surfaces and substituents have important implications for the design of new phosphate ester molecules for use as VPLs and antiwear additives.

\section{Acknowledgements}

J.P.E. and D.D. thank the Engineering and Physical Sciences Research Council (EPSRC) for grant EP/P030211/1 and Established Career Fellowship EP/N025954/1. C.A.L. thanks the EPSRC and Afton Chemical Corporation for Ph.D. funding via the Theory and Simulation of Materials-Centre for Doctoral Training (TSM-CDT) EP/L015579/1. A.K. and A.M. acknowledge support from the U.S. Army Research Laboratory under Cooperative Agreement No. W911NF-16-2-0121. The authors acknowledge the use of Imperial College London Research Computing Service (RCS). C.G. is supported by the European Union's Horizon 2020 research and innovation programme under the Marie Skłodowska-Curie grant agreement No. 744027. C.G.'s DFT calculations were facilitated by a grant from the Swiss National Supercomputing Centre (CSCS) under project ID s870. All data reported in the manuscript can be obtained by emailing the corresponding author or tribology@imperial.ac.uk.

\section{Author Contributions}

J.P.E., D.D., J.D.M, and J.E.R. designed the study, A.K. and A.M. parameterised the ReaxFF force field and provided some of the initial configurations and post-processing scripts which were modified by C.A.L., J.P.E. set up and ran the MD simulations, C.A.L. post-processed the data and produced the figures, C.G. performed the DFT calculations, J.P.E. wrote the original version of the manuscript which was subsequently revised by all authors.

\section{ORCID}

J.P.E. 0000-0001-5110-6970

C.A.L. 0000-0002-4508-0644

C.G. $0000-0002-3376-6374$

A.K. 0000-0002-9061-9370

J.D.M. 0000-0002-0231-0358

A.M. 0000-0003-2017-6081

D.D. 0000-0002-5518-499X 


\section{Supporting Information}

Additional figures showing the change in the total number of bonds within the phosphate ester molecules during the heating simulations (Fig. S1 and Fig. S2). ReaxFF parameter file. Videos of some representative thermal decomposition simulations are also provided: TNBP on $\mathrm{Fe}_{3} \mathrm{O}_{4}(001)$, TNBP on $\alpha-\mathrm{Fe}(110)$, and TNBP on $a-\mathrm{Fe}_{3} \mathrm{O}_{4}-\mathrm{OH}$.

\section{References}

(1) Taylor, R. I. Tribology and Energy Efficiency: From Molecules to Lubricated Contacts to Complete Machines. Faraday Discuss. 2012, 156, 361-382.

(2) McFadden, C.; Soto, C.; Spencer, N. D. Adsorption and Surface Chemistry in Tribology. Tribol. Int. 1998, 30 (12), 881-888.

(3) Spikes, H. The History and Mechanisms of ZDDP. Tribol. Lett. 2004, 17 (3), 469-489.

(4) Bodek, K. M.; Wong, V. V. The Effects of Sulfated Ash, Phosphorus and Sulfur on Diesel Aftertreatment Systems-A Review. SAE Tech. Pap. 2007, 2007-01-1922.

(5) Spikes, H. Low- and Zero-Sulphated Ash, Phosphorus and Sulphur Anti-Wear Additives for Engine Oils. Lubr. Sci. 2008, 20, 103-136.

(6) Johnson, D.; Hils, J. Phosphate Esters, Thiophosphate Esters and Metal Thiophosphates as Lubricant Additives. Lubricants 2013, 1 (4), 132-148.

(7) Devlin, M. T.; Guevremont, J.; Sheets, R.; Loper, J.; Guinther, G.; Thompson, K.; Jao, T.-C. Effect of Metal-Free Phosphorus Anti-Wear Compounds on Passenger Car Emissions and Fuel Economy. Lubr. Sci. 2008, 20, 151-161.

(8) Guan, B.; Pochopien, B. A.; Wright, D. S. The Chemistry, Mechanism and Function of Tricresyl Phosphate (TCP) as an Anti-Wear Lubricant Additive. Lubr. Sci. 2016, 28, 257-265.

(9) Graham, E. E.; Klaus, E. E. Lubrication from the Vapor Phase at High Temperatures. ASLE Trans. 1986, 29 (2), 229-234.

(10) Beeck, O.; Givens, J. W.; Williams, E. C. On the Mechanism of Boundary Lubrication II. Wear Prevention by Addition Agents. Proc. R. Soc. London, Ser. A 1940, 177 (968), 103-118.

(11) Barcroft, F. T.; Daniel, S. G. The Action of Neutral Organic Phosphates as EP Additives. J. Basic Eng. 1965, 87 (3), 761-767. 
(12) Godfrey, D. The Lubrication Mechanism of Tricresyl Phosphate on Steel. ASLE Trans. 1965, $8(1), 1-11$.

(13) Faut, O. D.; Wheeler, D. R. On the Mechanism of Lubrication by Tricresylphosphate (TCP)-the Coefficient of Friction as a Function of Temperature for TCP on M-50 Steel. ASLE Trans. 1983, 26 (3), 344-350.

(14) Gauthier, A.; Montes, H.; Georges, J. M. Boundary Lubrication with Tricresylphosphate (TCP). Importance of Corrosive Wear. ASLE Trans. 1982, 25 (4), 445-455.

(15) Saba, C. S.; Forster, N. H. Reactions of Aromatic Phosphate Esters with Metals and Their Oxides. Tribol. Lett. 2002, 12 (2), 135-146.

(16) Johnson, D. W.; Morrow, S.; Forster, N. H.; Saba, C. S. Vapor-Phase Lubrication: Reaction of Phosphate Ester Vapors with Iron and Steel. Chem. Mater. 2002, 14 (9), 3767-3775.

(17) Acharya, B.; Pardue, T. N.; Avva, K. S.; Krim, J. In Situ, Real Time Studies of Thermal Reaction Film Formation Temperatures for Iron and 304SS Surfaces Immersed in 5\% Tricresyl Phosphate in Base Oil. Tribol. Int. 2018, 126, 106-115.

(18) Bieber, H. E.; Klaus, E. E.; Tewksbury, E. J. A Study of Tricresyl Phosphate as an Additive for Boundary Lubrication. ASLE Trans. 1968, 11 (2), 155-161.

(19) Goldblatt, I. L.; Appeldoorn, J. K. The Antiwear Behavior of TCP in Different Atmospheres and Different Base Stocks. ASLE Trans. 1970, 13 (3), 203-214.

(20) Cho, L.; Klaus, E. E. Oxidative Degradation of Phosphate Esters. ASLE Trans. 1981, $24(1), 119-124$.

(21) Dörr, N.; Brenner, J.; Ristić, A.; Ronai, B.; Besser, C.; Pejaković, V.; Frauscher, M. Correlation Between Engine Oil Degradation, Tribochemistry, and Tribological Behavior with Focus on ZDDP Deterioration. Tribol. Lett. 2019, 67, 62.

(22) Yamamoto, Y.; Hirano, F. Scuffing Resistance of Phosphate Esters. Wear 1978, 50, 343-348.

(23) Yamamoto, Y.; Hirano, F. The Effect of the Addition of Phosphate Esters to Paraffinic Base Oils on Their Lubricating Performance under Sliding Conditions. Wear 1982, 78 (3), 285-296.

(24) Davey, W. Extreme Pressure Lubricants - Phosphorous Compounds as Additives. Ind. Eng. Chem. 1950, 42 (9), 1841-1847. 
(25) Sung, D.; Gellman, A. J. The Surface Chemistry of Alkyl and Arylphosphate Vapor Phase Lubricants on Fe Foil. Tribol. Int. 2002, 35 (9), 579-590.

(26) Sung, D.; Gellman, A. J. Thermal Decomposition of Tricresylphosphate Isomers on Fe. Tribol. Lett. 2002, 13 (1), 9-14.

(27) Gao, F.; Kotvis, P. V; Stacchiola, D.; Tysoe, W. T. Reaction of Tributyl Phosphate with Oxidized Iron: Surface Chemistry and Tribological Significance. Tribol. Lett. 2005, 18 (3), 377-384.

(28) Ewen, J. P.; Heyes, D. M.; Dini, D. Advances in Nonequilibrium Molecular Dynamics Simulations of Lubricants and Additives. Friction 2018, 6 (4), 349-386.

(29) Osei-Agyemang, E.; Berkebile, S.; Martini, A. Decomposition Mechanisms of AntiWear Lubricant Additive Tricresyl Phosphate on Iron Surfaces Using DFT and Atomistic Thermodynamic Studies. Tribol. Lett. 2018, 66, 48.

(30) Koyama, M.; Hayakawa, J.; Onodera, T.; Ito, K.; Tsuboi, H.; Endou, A.; Kubo, M.; Del Carpio, C. A.; Miyamoto, A. Tribochemical Reaction Dynamics of Phosphoric Ester Lubricant Additive by Using a Hybrid Tight-Binding Quantum Chemical Molecular Dynamics Method. J. Phys. Chem. B 2006, 110 (35), 17507-17511.

(31) Loehlé, S.; Righi, M. Ab Initio Molecular Dynamics Simulation of Tribochemical Reactions Involving Phosphorus Additives at Sliding Iron Interfaces. Lubricants 2018, $6(2), 31$.

(32) Loehlé, S.; Righi, M. C. First Principles Study of Organophosphorus Additives in Boundary Lubrication Conditions: Effects of Hydrocarbon Chain Length. Lubr. Sci. 2017, 29, 485-491.

(33) van Duin, A. C. T.; Dasgupta, S.; Lorant, F.; Goddard III, W. A. ReaxFF: A Reactive Force Field for Hydrocarbons. J. Phys. Chem. A 2001, 105 (41), 9396-9409.

(34) Plimpton, S. J.; Thompson, A. P. Computational Aspects of Many-Body Potentials. MRS Bull. 2012, 37 (5), 513-521.

(35) Senftle, T. P.; Hong, S.; Islam, M. M.; Kylasa, S. B.; Zheng, Y.; Shin, Y. K.; Junkermeier, C.; Engel-Herbert, R.; Janik, M. J.; Aktulga, H. M.; et al. The ReaxFF Reactive Force-Field: Development, Applications and Future Directions. npj Comput. Mater. 2016, 2, 15011.

(36) Khajeh, A.; Hu, X.; Mohammadtabar, K.; Shin, Y. K.; van Duin, A. C. T.; Berkebile, S.; Martini, A. Statistical Analysis of Tri-Cresyl Phosphate Conversion on an Iron Oxide 
Surface Using Reactive Molecular Dynamics Simulations. J. Phys. Chem. C 2019, 123, 12886-12893.

(37) Khajeh, A.; Krim, J.; Martini, A. Synergistic Effect of Nanodiamonds on the Adsorption of Tricresyl Phosphate on Iron Oxide Surfaces. Appl. Phys. Lett. 2019, 114, 171602.

(38) Bahlakeh, G.; Ghaffari, M.; Saeb, M. R.; Ramezanzadeh, B.; De Proft, F.; Terryn, H. A Close-up of the Effect of Iron Oxide Type on the Interfacial Interaction between Epoxy and Carbon Steel: Combined Molecular Dynamics Simulations and Quantum Mechanics. J. Phys. Chem. C 2016, 120 (20), 11014-11026.

(39) Chia, C.-L.; Siperstein, F. R.; Filip, S. Liquid Adsorption of Organic Compounds on Hematite $\alpha-F e 2 O 3$ Using ReaxFF. Langmuir 2017, 33, 11257-11263.

(40) Parkinson, G. S. Iron Oxide Surfaces. Surf. Sci. Rep. 2016, 71, 272-365.

(41) Chan-Rosado, G.; Pech-Canul, M. A. Influence of Native Oxide Film Age on the Passivation of Carbon Steel in Neutral Aqueous Solutions with a Dicarboxylic Acid. Corros. Sci. 2019, 153, 19-31.

(42) Evans, R. D.; More, K. L.; Darragh, C. V.; Nixon, H. P. Transmission Electron Microscopy of Boundary-Lubricated Bearing Surfaces. Part I: Mineral Oil Lubricant. Tribol. Trans. 2004, 47 (3), 430-439.

(43) Evans, R. D.; More, K. L.; Darragh, C. V.; Nixon, H. P. Transmission Electron Microscopy of Boundary-Lubricated Bearing Surfaces. Part II: Mineral Oil Lubricant with Sulfur- and Phosphorus-Containing Gear Oil Additives. Tribol. Trans. 2005, 48 (3), 299-307.

(44) Lancaster, J. K. A Review of the Influence of Environmental Humidity and Water on Friction, Lubrication and Wear. Tribol. Int. 1990, 23 (6), 371-389.

(45) Parkinson, G. S.; Novotný, Z.; Jacobson, P.; Schmid, M.; Diebold, U. Room Temperature Water Splitting at the Surface of Magnetite. J. Am. Chem. Soc. 2011, 133 (32), 12650-12655.

(46) Fischer, T. E. Tribochemistry. Ann. Rev. Mater. Sci. 1988, 18, 303-323.

(47) Błoński, P.; Kiejna, A. Structural, Electronic, and Magnetic Properties of BCC Iron Surfaces. Surf. Sci. 2007, 601 (1), 123-133.

(48) Pentcheva, R.; Wendler, F.; Meyerheim, H. L.; Moritz, W.; Jedrecy, N.; Scheffler, M. Jahn-Teller Stabilization of a "Polar" Metal Oxide Surface: Fe304(001). Phys. Rev. Lett. 2005, 94 (12), 126101. 
(49) Plaza, S. The Adsorption of Zinc Dibutyldithiophosphates on Iron and Iron Oxide Powders. ASLE Trans. 1987, 30 (2), 233-240.

(50) Stukowski, A. Visualization and Analysis of Atomistic Simulation Data with OVITO-the Open Visualization Tool. Model. Simul. Mater. Sci. Eng. 2010, 18, 015012.

(51) Plimpton, S. Fast Parallel Algorithms for Short-Range Molecular-Dynamics. J. Comput. Phys. 1995, 117 (1), 1-19.

(52) Nosé, S. A Molecular-Dynamics Method for Simulations in the Canonical Ensemble. Mol. Phys. 1984, 52 (2), 255-268.

(53) Hoover, W. G. Canonical Dynamics: Equilibrium Phase-Space Distributions. Phys. Rev. A 1985, 31 (3), 1695-1697.

(54) Chenoweth, K.; van Duin, A. C. T.; Goddard III, W. A. ReaxFF Reactive Force Field for Molecular Dynamics Simulations of Hydrocarbon Oxidation. J. Phys. Chem. A 2008, 112 (5), 1040-1053.

(55) Aktulga, H. M.; Fogarty, J. C.; Pandit, S. A.; Grama, A. Y. Parallel Reactive Molecular Dynamics: Numerical Methods and Algorithmic Techniques. Parallel Comput. 2012, 38 (4-5), 245-259.

(56) Rappe, A. K.; Goddard III, W. A. Charge Equilibration for Molecular Dynamics Simulations. J. Phys. Chem. 1991, 95, 3358-3363.

(57) Nakano, A. Parallel Multilevel Preconditioned Conjugate-Gradient Approach to Variable-Charge Molecular Dynamics. Comput. Phys. Commun. 1997, 104 (1-3), 5969.

(58) Aryanpour, M.; van Duin, A. C. T.; Kubicki, J. D. Development of a Reactive Force Field for Iron-Oxyhydroxide Systems. J. Phys. Chem. A 2010, 114 (21), 6298-6307.

(59) Zou, C.; van Duin, A. C. T.; Sorescu, D. C. Theoretical Investigation of Hydrogen Adsorption and Dissociation on Iron and Iron Carbide Surfaces Using the ReaxFF Reactive Force Field Method. Top. Catal. 2012, 55 (5-6), 391-401.

(60) Verlackt, C. C. W.; Neyts, E. C.; Jacob, T.; Fantauzzi, D.; Golkaram, M.; Shin, Y. K.; van Duin, A. C. T.; Bogaerts, A. Atomic-Scale Insight into the Interactions between Hydroxyl Radicals and DNA in Solution Using the ReaxFF Reactive Force Field. New J. Phys. 2015, 17, 103005.

(61) Handrigan, S. M.; Morrissey, L. S.; Nakhla, S. Investigating Various Many-Body Force Fields for Their Ability to Predict Reduction in Elastic Modulus Due to Vacancies 
Using Molecular Dynamics Simulations. Mol. Simul. 2019, 45 (16), 1341-1352.

(62) Zhang, L.; van Duin, A. C. T.; Zybin, S. V.; Goddard III, W. A. Thermal Decomposition of Hydrazines from Reactive Dynamics Using the ReaxFF Reactive Force Field. J. Phys. Chem. B 2009, 113 (31), 10770-10778.

(63) Chaudret, R.; Bick, A.; Krokidis, X. Theoretical Modeling of Thermal Decomposition of Methylnaphthalene Derivatives: Influence of Substituents. Energy \& Fuels 2016, 30 (8), 6817-6821.

(64) Mueller, J. E.; van Duin, A. C. T.; Goddard III, W. A. Application of the ReaxFF Reactive Force Field to Reactive Dynamics of Hydrocarbon Chemisorption and Decomposition. J. Phys. Chem. C 2010, 114 (12), 5675-5685.

(65) Zou, C.; van Duin, A. Investigation of Complex Iron Surface Catalytic Chemistry Using the ReaxFF Reactive Force Field Method. JOM 2012, 64 (12), 1426-1437.

(66) Yamada, T.; Phelps, D. K.; van Duin, A. C. T. First Principle and ReaxFF Molecular Dynamics Investigations of Formaldehyde Dissociation on Fe(100) Surface. J. Comput. Chem. 2013, 34 (23), 1982-1996.

(67) Shin, Y. K.; Kwak, H.; Vasenkov, A. V; Sengupta, D.; van Duin, A. C. T. Development of a ReaxFF Reactive Force Field for $\mathrm{Fe} / \mathrm{Cr} / \mathrm{O} / \mathrm{S}$ and Application to Oxidation of Butane over a Pyrite-Covered Cr2O3 Catalyst. ACS Catal. 2015, 5, 7226-7236.

(68) Kresse, G.; Hafner, J. Ab Initio Molecular Dynamics for Liquid Metals. Phys. Rev. B 1993, 47 (1), 558.

(69) Kresse, G.; Furthmuller, J. Efficiency of Ab-Initio Total Energy Calculations for Metals and Semiconductors Using a Plane-Wave Basis Set. Comput. Mater. Sci. 1996, 6, $15-50$.

(70) Kresse, G.; Furthmuller, J. Efficient Iterative Schemes for Ab Initio Total-Energy Calculations Using a Plane-Wave Basis Set. Phys. Rev. B 1996, 54 (16), 11169.

(71) Kresse, G.; Joubert, D. From Ultrasoft Pseudopotentials to the Projector AugmentedWave Method. Phys. Rev. B. 1999, 59 (3), 1758-1775.

(72) Perdew, J. P.; Burke, K.; Ernzerhof, M. Generalized Gradient Approximation Made Simple. Phys. Rev. Lett. 1996, 77 (18), 3865-3868.

(73) Klimeš, J.; Bowler, D. R.; Michaelides, A. Van Der Waals Density Functionals Applied to Solids. Phys. Rev. B 2011, 83, 195131. 
(74) Gattinoni, C.; Michaelides, A. Understanding Corrosion Inhibition with van Der Waals DFT Methods: The Case of Benzotriazole. Faraday Discuss. 2015, 180, 439-458.

(75) Gattinoni, C.; Ewen, J. P.; Dini, D. Adsorption of Surfactants on $\alpha-F e 2 O 3(0001):$ A Density Functional Theory Study. J. Phys. Chem. C 2018, 122 (36), 20817-20826.

(76) Monkhorst, H. J.; Pack, J. D. Special Points for Brillouin-Zone Integrations. Phys. Rev. B 1976, 13 (12), 5188-5192.

(77) Makov, G.; Payne, M. Periodic Boundary Conditions in Ab Initio Calculations. Phys. Rev. B 1995, 51 (7), 4014-4022.

(78) Henkelman, G.; Uberuaga, B. P.; Jónsson, H. A Climbing Image Nudged Elastic Band Method for Finding Saddle Points and Minimum Energy Paths. J. Chem. Phys. 2000, $113,9901$.

(79) Tamura, H.; Tanaka, A.; Ito, M. Mechanism of Hydroxylation of Metal Oxide Surfaces. J. Colloid Interface Sci. 2001, 243, 202-207.

(80) Blunt, T. J.; Kotvis, P. V.; Tysoe, W. T. Determination of Interfacial Temperatures under Extreme Pressure Conditions. Tribol. Lett. 1996, 2 (3), 221-230.

(81) Wellendorff, J.; Silbaugh, T. L.; Garcia-Pintos, D.; Nørskov, J. K.; Bligaard, T.; Studt, F.; Campbell, C. T. A Benchmark Database for Adsorption Bond Energies to Transition Metal Surfaces and Comparison to Selected DFT Functionals. Surf. Sci. 2015, 640, 36-44.

(82) Hu, X.; Schuster, J.; Schulz, S. E. Multiparameter and Parallel Optimization of ReaxFF Reactive Force Field for Modeling the Atomic Layer Deposition of Copper. J. Phys. Chem. C 2017, 121 (50), 28077-28089.

(83) Rivera de la Cruz, J. G.; Sabbe, M. K.; Reyniers, M. F. First Principle Study on the Adsorption of Hydrocarbon Chains Involved in Fischer-Tropsch Synthesis over Iron Carbides. J. Phys. Chem. C 2017, 121 (45), 25052-25063.

(84) Abdelmaksoud, M.; Bender, J. W.; Krim, J. Nanotribology of a Vapor-Phase Lubricant: A Quartz Crystal Microbalance Study of Tricresylphosphate (TCP) Uptake on Iron and Chromium. Tribol. Lett. 2002, 13 (3), 179-186.

(85) Sattler, J. J. H. B.; Ruiz-Martinez, J.; Santillan-Jimenez, E.; Weckhuysen, B. M. Catalytic Dehydrogenation of Light Alkanes on Metals and Metal Oxides. Chem. Rev. 2014, 114 (20), 10613-10653.

(86) Jones, R. B.; Coy, R. C. The Chemistry of the Thermal Degradation of Zinc 
Dialkyldithiophosphate Additives. ASLE Trans. 1981, 24 (1), 91-97.

(87) Wheeler, D. R.; Faut, O. D. The Adsorption and Thermal Decomposition of Tricresylphosphate (TCP) on Iron and Gold. Appl. Surf. Sci. 1984, 18 (1-2), 106-122.

(88) Holbert, A. W.; Batteas, J. D.; Wong-Foy, A.; Rufael, T. S.; Friend, C. M. Passivation of Fe (110) via Phosphorus Deposition: The Reactions of Trimethylphosphite. Surf. Sci. 1998, 401 (3), 437-443.

(89) Liang, J.; Zhang, Q.; Wu, H.; Meng, G.; Tang, Q.; Wang, Y. Iron-Based Heterogeneous Catalysts for Epoxidation of Alkenes Using Molecular Oxygen. Catal. Commun. 2004, 5 (11), 665-669.

(90) Zhang, J.; Ewen, J. P.; Ueda, M.; Wong, J. S. S.; Spikes, H. A. Mechanochemistry of Zinc Dialkyldithiophosphate on Steel Surfaces under Elastohydrodynamic Lubrication Conditions. ACS Appl. Mater. Interfaces 2020, 12 (5), 6662-6676.

(91) Rowe, C. N.; Dickert, J. J. The Relation of Antiwear Function to Thermal Stability and Structure for Metal: O,O-Dialkylphosphorodithioates. ASLE Trans. 1967, 10 (1), 8590.

(92) Fujita, H.; Glovnea, R. P.; Spikes, H. A. Study of Zinc Dialkydithiophosphate Antiwear Film Formation and Removal Processes, Part I: Experimental. Tribol. Trans. 2005, 48 (4), 558-566.

(93) Topolovec-Miklozic, K.; Forbus, T. R.; Spikes, H. A. Film Thickness and Roughness of ZDDP Antiwear Films. Tribol. Lett. 2007, 26 (2), 161-171.

(94) Ferguson, S.; Johnson, J.; Gonzales, D.; Hobbs, C.; Allen, C.; Williams, S. Analysis of ZDDP Content and Thermal Decomposition in Motor Oils Using NAA and NMR. Phys. Procedia 2015, 66, 439-444.

(95) Spikes, H. A. Additive-Additive and Additive-Surface Interactions in Lubrication. Lubr. Sci. 1989, 2 (1), 3-23.

(96) Fuller, M.; Yin, Z.; Kasrai, M.; Bancroft, G. M.; Yamaguchi, E. S.; Ryason, P. R.; Wiliermet, P. A.; Tan, K. H. Chemical Characterization of Tribochemical and Thermal Films Generated from Neutral and Basic ZDDPs Using X-Ray Absorption Spectroscopy. Tribol. Int. 1997, 30 (4), 305-315.

(97) Berkani, S.; Dassenoy, F.; Minfray, C.; Martin, J. M.; Cardon, H.; Montagnac, G.; Reynard, B. Structural Changes in Tribo-Stressed Zinc Polyphosphates. Tribol. Lett. 2013, 51 (3), 489-498. 
(98) Ribas-Arino, J.; Marx, D. Covalent Mechanochemistry: Theoretical Concepts and Computational Tools with Applications to Molecular Nanomechanics. Chem. Rev. 2012, 112, 5412-5487.

(99) Yue, D. C.; Ma, T. B.; Hu, Y. Z.; Yeon, J.; van Duin, A. C. T.; Wang, H.; Luo, J. B. Tribochemistry of Phosphoric Acid Sheared between Quartz Surfaces: A Reactive Molecular Dynamics Study. J. Phys. Chem. C 2013, 117 (48), 25604-25614.

(100) Yeon, J.; He, X.; Martini, A.; Kim, S. H. Mechanochemistry at Solid Surfaces: Polymerization of Adsorbed Molecules by Mechanical Shear at Tribological Interfaces. ACS Appl. Mater. Interfaces 2017, 9, 3142-3148.

(101) Mohammadtabar, K.; Eder, S. J.; Dörr, N.; Martini, A. Heat-, Load-, and Shear-Driven Reactions of Di-Tert-Butyl Disulfide on Fe(100). J. Phys. Chem. C 2019, 123, 1968819692.

\section{Table of Contents Graphic}

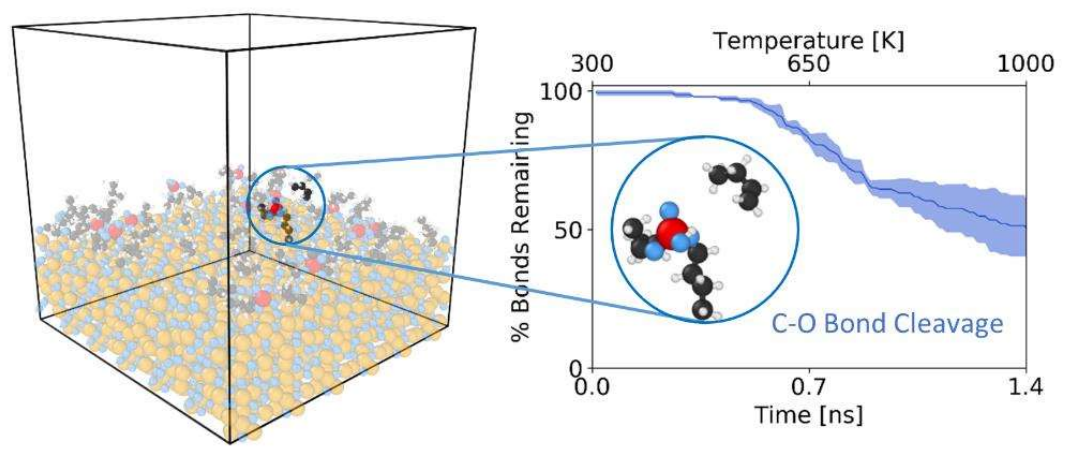

